\title{
MICROLOCAL REGULARITY THEOREMS FOR NONSMOOTH PSEUDODIFFERENTIAL OPERATORS AND APPLICATIONS TO NONLINEAR PROBLEMS
}

\author{
$\mathrm{BY}$ \\ MICHAEL BEALS ${ }^{1}$ AND MICHAEL REED ${ }^{2}$
}

\begin{abstract}
The authors develop a calculus of pseudodifferential operators with nonsmooth coefficients in order to study the regularity of solutions to linear equations $P(x, D) u=f$. The regularity theorems are similar to those of Bony, but the calculus and the methods of proof are quite different. We apply the linear results to study the regularity properties of solutions to quasilinear partial differential equations.
\end{abstract}

Introduction. In this paper we continue our study of the regularity of solutions of $P(x, D) u=f$ where $P(x, D)$ is a nonsmooth pseudodifferential operator. In $[\mathbf{1}]$, a simple commutator lemma and Rauch's Lemma allowed us to prove a microlocal propagation of regularity theorem, analogous to Hörmander's theorem, in the case where the highest order term of $P(x, D)$ is a smooth classical pseudodifferential operator. We then showed how this result could be applied to semilinear partial differential equations. In this paper we develop a calculus to handle the situation when the highest order term of $P(x, D)$ is nonsmooth and apply the results to quasilinear partial differential equations. Our results for quasilinear equations are similar to those of Bony [4], but our methods are quite different. Bony uses the Littlewood-Paley theory and the machinery of paradifferential operators. We develop a simple calculus for certain classes of nonsmooth symbols which is analogous to the classical calculus of pseudodifferential operators except that, because of limited regularity, expansions have only finitely many terms. We believe that the calculus and linear theorems which we develop will be useful in a variety of other contexts.

In $\S 1$ we introduce a class of nonsmooth symbols and develop a partial calculus. Very general symbol classes typically yield weak theorems which do not give very much information in applications. Very restricted symbol classes may handle specific applications but are unlikely to be of general use. We have attempted to avoid both of these pitfalls by defining symbol classes which are simple, which yield the information we want in our nonlinear applications, but which are sufficiently general that they may be useful in other contexts. They are the microlocal analogue of classes of symbols with "rough coefficients" which have been used previously in the context of elliptic equations; see for example Beals, Fefferman and Grossman $[2]$.

Received by the editors April 19, 1983 and, in revised form, October 10, 1983.

1980 Mathematics Subject Classification. Primary 35S05, 35L75; Secondary 35L70, 35J60.

${ }^{1}$ Research partially supported by NSF Grant \#MCS-8201281.

${ }^{2}$ Research partially supported by NSF Grant \#MCS-8201258. 
In $§ 2$, we apply the calculus to give an easy proof of a regularity theorem for elliptic operators with nonsmooth coefficients. The disadvantage of the result is that we can only deal with coefficients in appropriate $H^{s}$ spaces for $s>n / 2$ and the hypotheses and conclusions are all in terms of the $L^{2}$ category. The advantage, besides the ease of proof, is that our theorem applies in all space dimensions and to elliptic operators of any order. For comparisons with the standard theory, see Gilbarg and Trudinger [6].

In [1], our proof of the analogue of Hörmander's theorem on the propagation of singularities followed the outline in Hörmander [7] and Nirenberg [9]. That method was possible because the highest order symbol was smooth, so that a microlocal cutoff $b_{0}(x, \xi) \in S_{1,0}^{0}$ could be found such that the principal symbol of the commutator $\left[b_{0}, p_{m}(x, D)\right]$ vanished. If $p_{m}$ is nonsmooth then a smooth $b_{0}$ will not satisfy this restriction. However, as noted by Bony, the strong Gårding inequality allows the choice of a $b_{0}$ satisfying weaker conditions. We thus begin $\S 3$ by proving the strong Gårding inequality for symbols in the classes introduced in $\S 1$. We then follow the general approach of Hörmander [8] in proving the microlocal propagation of regularity results. The key technical tool is the partial calculus developed in $\S 1$.

In $\S 4$ we show how the linear results of $\S 1,2$ and 3 can be used in nonlinear problems. First, we apply them to general quasilinear partial differential equations (Theorem 4.1). Then we consider the example

$$
u_{t t}-\sum b_{i j}(\nabla u) \partial_{i} \partial_{j} u=0
$$

in detail. Finally, we treat the coupled elliptic-hyperbolic quasilinear system

$$
\begin{gathered}
\sum a_{i j}(u) \partial_{i} \partial_{j} v=g(u, v), \\
u_{t t}-\sum b_{i j}(v) \partial_{i} \partial_{j} u=f(u, v) .
\end{gathered}
$$

The analysis of these examples makes clear the necessity of the microlocal hypotheses and conclusions in the linear theorems developed in this paper.

It is appropriate to conclude this Introduction with some general remarks about the usefulness of the methods of this paper in nonlinear problems. First of all, it is known that in a variety of special situations singularities do not interact and spread to the extent permitted by the general theorems of this paper. These special situations include: one space dimension, Rauch and Reed [12]; special initial data, Bony [3], Rauch and Reed [13]; special structural conditions on nonlinear terms, Rauch and Reed [11].

Secondly, the great drawback of the Fourier analytic methods of this paper in nonlinear problems is that to use the methods solutions must be in $H^{s}$ for appropriate $s>n / 2$. Fourier analysis takes place most naturally in the $L^{2}$ category and it is difficult to build a general machine for controlling $\left(u^{2}\right)^{-}=\hat{u} * \hat{u}$ if $\hat{u}$ is not in $L^{1}$ (hence $s>n / 2$ ). But many questions about nonlinear partial differential equations are naturally posed in non- $L^{2}$ categories and many interesting phenomena (like shocks) guarantee that some important solutions will be in $H^{s}$ for $s<n / 2$. These questions and solutions cannot be analyzed by the methods of this paper. We can only treat relatively weak singularities.

Nevertheless, there are many interesting phenomena in the creation, interaction, and spreading of these weak singularities. We have tried to show, in this paper and 
its predecessor [1] that the ideas of Fourier analysis and classical pseudodifferential operators can be extended naturally to nonsmooth categories of symbols and then applied to certain aspects of these nonlinear problems.

\section{A calculus for nonsmooth operators.}

DEFINITION 1.1. We will say that a distribution $u$ is in $H^{s} \cap H_{m l}^{r}\left(x_{0}, \xi_{0}\right)$ if there exist $\phi(x) \in C_{0}^{\infty}\left(\mathbf{R}^{n}\right)$ with $\phi\left(x_{0}\right) \neq 0$ and a conic neighborhood $K$ of $\xi_{0}$ in $\mathbf{R}^{n} \backslash\{0\}$ such that

$$
\langle\xi\rangle^{s}(\phi \mu) \widehat{\uparrow}(\xi) \in L^{2}\left(\mathbf{R}^{n}\right)
$$

and

$$
\langle\xi\rangle^{r} \chi_{K}(\xi)(\phi \mu)^{\wedge}(\xi) \in L^{2}\left(\mathbf{R}^{n}\right) .
$$

If $\gamma$ is a closed set in $T^{*}\left(\mathbf{R}^{n}\right) \backslash 0$, we shall say that $u \in H^{s} \cap H_{m l}^{r}(\gamma)$ if $u \in$ $H^{s} \cap H_{m l}^{r}(x, \xi)$ for all $(x, \xi) \in \gamma$. We always set $\langle\xi\rangle^{2} \equiv 1+\sum \xi_{i}^{2}$.

Nonsmooth operators of the form $a(x) p(x, D)$, where $a(x) \in H^{s} \cap H_{m l}^{r}(\gamma)$ and $p(x, \xi) \in S_{1,0}^{m}$ is a smooth symbol, are typical of those arising in nonlinear applications. If $a(x) \in H^{s+k} \cap H_{m l}^{r+k}(\gamma)$ and $p(x, D)$ is a partial differential operator of order $k$, then the composed operator $p(x, D) \circ a(x)$ has a symbol with the finite expansion $\sum_{|\alpha| \leq k}(1 / \alpha !) D_{x}^{\alpha} a(x) \partial_{\xi}^{\alpha} p(x, \xi)$, which is a sum of symbols with coefficients in $H^{s+k-|\alpha|} \cap H_{m l}^{r+k-|\alpha|}(\gamma)$. But if $p(x, \xi)$ is pseudodifferential, the usual complete asymptotic expansion $p \circ a(x, \xi) \sim \sum_{\alpha}(1 / \alpha !) D_{x}^{\alpha} a(x) \partial_{\xi}^{\alpha} p(x, \xi)$ cannot be taken, since for $|\alpha|>k$ the coefficients no longer have the desired smoothness of at least $H^{s} \cap H_{m l}^{r}(\gamma)$. Therefore we break off the expansion at step $k$, and set

$$
r(x, \xi)=p \circ a(x, \xi)-\sum_{|\alpha|<k} \frac{1}{\alpha !} D_{x}^{\alpha} a(x) \partial_{\xi}^{\alpha} p(x, \xi) .
$$

Such remainders will not in general be of the form $a(x) p(x, \xi)$. Thus we define a symbol class (with very weak hypotheses on the behavior in $\xi$ ) which will include such remainders.

DEFINITION 1.2. $S^{m ; s, r}(\gamma)$ is the collection of symbols $a(x, \xi)$, smooth in $\xi$, such that

$$
a(x, \xi) /\langle\xi\rangle^{m} \in H^{s} \cap H_{m l}^{r}(\gamma)
$$

as a function of $x$, uniformly in $\xi$. In other words, for each $\left(x_{0}, \eta_{0}\right) \in \gamma$ there exist $\phi(x) \in C_{0}^{\infty}\left(\mathbf{R}^{n}\right)$ with $\phi\left(x_{0}\right) \neq 0$ and a conic neighborhood $K$ of $\eta_{0}$ such that

$$
\begin{aligned}
& \langle\eta\rangle^{s}(\phi a)^{\wedge}(\eta, \xi) /\langle\xi\rangle^{m} \in L^{2}(d \eta) \text { and } \\
& \langle\eta\rangle^{r} \chi_{K}(\eta)(\phi a)^{\wedge}(\eta, \xi) /\langle\xi\rangle^{m} \in L^{2}(d \eta)
\end{aligned}
$$

with norms independent of $\xi$.

Classes of nonsmooth symbols with local rather than microlocal assumptions in $x$ and conditions on $\xi$ derivatives are used, for example, in Taylor [14, Chapter IV, $\S 5]$. But the minimal hypotheses of Definition 1.2 are sufficient for many purposes, in particular for proving that operators of order zero are bounded on $H^{s} \cap H_{m l}^{r}(\gamma)$ for appropriate $s$ and $r$. 
THEOREM 1.3. Let $n / 2<s \leq r<2 s-n / 2$ and suppose $a(x, \xi) \in S^{m ; s, r}(\gamma)$, $0 \leq m \leq s$. Then $a(x, D)$ is a bounded operator from $H^{s} \cap H_{m l}^{r}(\gamma)$ to $H^{s-m} \cap$ $H_{m l}^{r-m}(\gamma)$.

LEMMA 1.4. Suppose that

$$
C_{g}^{2} \equiv \sup _{\xi} \int|g(\eta, \xi)|^{2} d \eta<\infty
$$

and

$$
C_{G}^{2} \equiv \sup _{\eta} \int|G(\eta, \xi)|^{2} d \xi<\infty
$$

For $h \in L^{2}$, define

$$
(T h)(\eta)=\int G(\eta, \xi) g(\eta-\xi, \xi) h(\xi) d \xi .
$$

Then $\|T h\|_{2} \leq C_{g} C_{G}\|h\|_{2}$.

Proof. Simply write $\|T h\|_{2}=\sup _{\|f\|_{2} \leq 1}\left|\int f(T h)(\eta) d \eta\right|$, interchange integrals and use the Schwarz inequality.

Proof OF THEOREM 1.3. For $u \in H^{s} \cap H_{m l}^{r}(\gamma)$, we can assume that $u$ and $a(x, \xi)$ have compact support in $x$ near $x_{0}$, where $\left(x_{0}, \xi_{0}\right) \in \gamma$. Then,

$$
\begin{aligned}
a(x, D) u(x) & \equiv \int e^{i x \cdot \xi} a(x, \xi) \hat{u}(\xi) d \xi, \\
(a u)^{\Upsilon}(\eta) & =\int \hat{a} \cdot(\eta-\xi, \xi) \hat{u}(\xi) d \xi,
\end{aligned}
$$

where the hat on $a$ denotes the partial Fourier transform with respect to the first variable. Throughout the paper, $d \xi$ is normalized to absorb the factor $(2 \pi)^{-n}$. Since $a \in S^{m ; r, s}$,

$$
\hat{a}(\varsigma, \xi)=g(\varsigma, \xi)\langle\xi\rangle^{m} /\langle\varsigma\rangle^{s}
$$

where $\sup _{\xi} \int g^{2}(\varsigma, \xi) d \zeta<\infty$. Define $h=\langle\xi\rangle^{s} \hat{u}$. Then $h \in L^{2}$ and we have

$$
\langle\eta\rangle^{s-m}(a u)^{\wedge}(\eta)=\int G(\eta, \xi) g(\eta-\xi, \xi) h(\xi) d \xi
$$

where

$$
G(\eta, \xi)=\langle\eta\rangle^{s-m} /\langle\eta-\xi\rangle^{s}\langle\xi\rangle^{s-m} .
$$

Simple estimates and Hölder's inequality show that $\sup _{\eta} \int G(\eta, \xi)^{2} d \xi<\infty$. Thus, by Lemma 1.4, we conclude that $\langle\eta\rangle^{s-m}(a u)^{\wedge}(\eta) \in L^{2}$ which proves that $a(x, D) u$ $\in H^{s-m}$.

Now let $K$ be a conic neighborhood of $\xi_{0}$, where $\left(x_{0}, \xi_{0}\right) \in \gamma$, which is small enough so that the estimates in Definitions 1.1 and 1.2 hold for $u$ and $a$ on $K$. Let $\tilde{K}$ be a strictly smaller conic neighborhood (denoted $\tilde{K} \subset \subset K$ from now on). We must show

$$
\chi_{\tilde{K}}(\eta)\langle\eta\rangle^{r-m}(a u)^{-}(\eta) \in L^{2} .
$$


To do this, we set $\chi=\chi_{\tilde{K}}, \chi_{1}=\chi_{K}, \chi_{2}=\chi_{K^{c}}$ and write

$$
\begin{aligned}
\hat{a}(\zeta, \xi) & =\frac{\chi_{1}(\zeta) g_{1}(\zeta, \xi)\langle\xi\rangle^{m}}{\langle\zeta\rangle^{r}}+\frac{\chi_{2}(\zeta) g_{2}(\zeta, \xi)\langle\xi\rangle^{m}}{\langle\zeta\rangle^{s}} \\
\hat{u}(\xi) & =\frac{\chi_{1}(\xi) h_{1}(\xi)}{\langle\xi\rangle^{r}}+\frac{\chi_{2}(\xi) h_{2}(\xi)}{\langle\xi\rangle^{s}}
\end{aligned}
$$

where $g_{i} \in L^{2}(d \xi)$ uniformly in $\xi$ and $h_{i} \in L^{2}$. Thus,

$$
\chi(\eta)\langle\eta\rangle^{r-m}(a u)-(\eta)=\sum_{i, j=1,2} \int G_{i j}(\eta, \xi) g_{i}(\eta-\xi, \xi) h_{j}(\xi) d \xi
$$

where

$$
\begin{aligned}
& G_{11}(\eta, \xi)=\frac{\chi(\eta) \chi_{1}(\eta-\xi) \chi_{1}(\xi)\langle\eta\rangle^{r-m}}{\langle\eta-\xi\rangle^{r}\langle\xi\rangle^{r-m}} \\
& G_{12}(\eta, \xi)=\frac{\chi(\eta) \chi_{1}(\eta-\xi) \chi_{2}(\xi)\langle\eta\rangle^{r-m}}{\langle\eta-\xi\rangle^{r}\langle\xi\rangle^{s-m}} \\
& G_{21}(\eta, \xi)=\frac{\chi(\eta) \chi_{2}(\eta-\xi) \chi_{1}(\xi)\langle\eta\rangle^{r-m}}{\langle\eta-\xi\rangle^{s}\langle\xi\rangle^{r-m}} \\
& G_{22}(\eta, \xi)=\frac{\chi(\eta) \chi_{2}(\eta-\xi) \chi_{2}(\xi)\langle\eta\rangle^{r-m}}{\langle\eta-\xi\rangle^{s}\langle\xi\rangle^{s-m}}
\end{aligned}
$$

$G_{11}$ is handled just as $G$ was above. On $\operatorname{supp} G_{12}, \xi \in K^{c}$ and $\eta \in \tilde{K}$ so $\langle\eta-\xi\rangle \geq$ $c\langle\eta\rangle$. Therefore

$$
G_{12} \leq \frac{c}{\langle\eta-\xi\rangle^{m}\langle\xi\rangle^{s-m}}
$$

Similarly, $\langle\xi\rangle \geq c\langle\eta\rangle$ on $\operatorname{supp} G_{21}$, so

$$
G_{21} \leq c /\langle\eta-\xi\rangle^{s} .
$$

Finally, $\langle\eta-\xi\rangle \geq c\langle\eta\rangle$ and $\langle\xi\rangle \geq c\langle\eta\rangle$ on $\operatorname{supp} G_{22}$ and since $r=2 s-n / 2-\varepsilon$ for some $\varepsilon>0$, we can estimate

$$
G_{22} \leq c /\langle\xi\rangle^{n / 2+\varepsilon}
$$

Thus, for each $i, j, \sup _{\eta} \int G_{i j}^{2}(\eta, \xi) d \xi<\infty$, so by Lemma 1.4, we conclude that (1.5) holds.

Theorem 1.3 contains as a special case (simply take $a(x, \xi)=a(x))$ the following result $[\mathbf{1 0}]$.

RAUCH'S LEMMA. $H^{s} \cap H_{m l}^{r}(\gamma)$ is an algebra for $n / 2<s \leq r<2 s-n / 2$.

Also, by writing $a(x, \xi)=\left(a(x, \xi)\langle\xi\rangle^{m}\right)\langle\xi\rangle^{-m}$, we see that Theorem 1.3 implies that the operators in $S^{0 ; s, r}(\gamma)$ are bounded on $H^{s-m} \cap H_{m l}^{r-m}(\gamma)$ for $0 \leq m \leq s$.

We now develop a partial calculus for the operators with symbols as in Definition 1.2. It is useful to keep in mind for motivation two cases which arise in applications. We often wish to compose with an operator of low order, say a microlocal cutoff of order zero or the operator corresponding to the reciprocal of a microlocally elliptic symbol. In this instance, it is useful to allow both of the symbols to be composed to be nonsmooth. On the other hand, in hyperbolic problems the symmetrization procedure makes it necessary to commute nonsmooth operators with operators like $\Lambda^{r}$, where $\Lambda$ has symbol $\langle\xi\rangle$. For these higher order operators it is only necessary to consider compositions in which the factor on the left has a smooth symbol. 
LEMMA 1.5. Let $n / 2<s \leq r<2 s-n / 2$, let $k \geq 0$ be an integer, and assume that $a(x, \xi) \in S^{m ; s+k, r+k}(\gamma)$ and $\partial_{\xi}^{\alpha} b(x, \xi) \in S^{k-|\alpha| ; s, r}(\gamma)$ for $|\alpha| \leq k$. Then $b(x, D) a(x, D)$ is an operator with symbol $b \circ a(x, \xi) \in S^{m+k ; s, r}(\gamma)$ satisfying

$$
\left\{b \circ a(x, \xi)-\sum_{|\alpha|<k} \frac{1}{\alpha !} \partial_{\xi}^{\alpha} b D_{x}^{\alpha} a(x, \xi)\right\} \in S^{m ; s, r}(\gamma) .
$$

PROOF.

$$
\begin{aligned}
b \circ a(x, D) u(x) & =\int e^{i x \cdot \xi} b(x, \xi)(a u)^{-}(\xi) d \xi \\
& =\iint e^{i x \cdot \xi} b(x, \xi) \hat{a}(\xi-\varsigma, \zeta) \hat{u}(\varsigma) d \varsigma d \xi
\end{aligned}
$$

Thus

$$
\begin{aligned}
(b \circ a u)^{-} & =\iint \hat{b}(\eta-\xi, \xi) \hat{a}(\xi-\varsigma, \zeta) \hat{u}(\zeta) d \xi d \zeta \\
& =\iint \hat{b}(\eta-\varsigma-\xi, \zeta+\xi) \hat{a}(\xi, \varsigma) \hat{u}(\zeta) d \xi d \zeta
\end{aligned}
$$

On the other hand,

$$
\partial_{\xi}^{\alpha} b D_{x}^{\alpha} a(x, D) u(x)=\int e^{i x \cdot \varsigma} \partial_{\zeta}^{\alpha} b D_{x}^{\alpha} a(x, \varsigma) \hat{u}(\varsigma) d \varsigma
$$

and

$$
\begin{aligned}
\left(\partial_{\xi}^{\alpha} b D_{x}^{\alpha} a u\right)^{\wedge}(\eta) & =\int\left(\partial_{\zeta}^{\alpha} b D_{x}^{\alpha} a\right)^{\wedge}(\eta-\varsigma, \zeta) \hat{u}(\zeta) d \zeta \\
& =\iint \partial_{\zeta}^{\alpha} b(\eta-\varsigma-\xi, \zeta) \xi^{\alpha} \hat{a}(\xi, \varsigma) \hat{u}(\zeta) d \xi d \zeta
\end{aligned}
$$

So, defining

$$
r(x, D) \equiv b \circ a(x, D)-\sum_{|\alpha|<k} \frac{1}{\alpha !} \partial_{\xi}^{\alpha} b D_{x}^{\alpha} a(x, D),
$$

we have

$$
\begin{aligned}
&(r u)^{-}(\eta)=\iint\left\{\hat{b}(\eta-\varsigma-\xi, \zeta+\xi)-\sum_{|\alpha|<k} \frac{1}{\alpha !} \xi^{\alpha}\left(\partial_{\zeta}^{\alpha} b\right)^{-}(\eta-\varsigma-\xi, \zeta)\right\} \\
& \times \hat{a}(\xi, \varsigma) \hat{u}(\varsigma) d \xi d \zeta .
\end{aligned}
$$

By Taylor's theorem, the right-hand side is

$$
\sum_{|\alpha|=k} c_{\alpha} \iint\left\{\int_{0}^{1}(1-t)^{k-1}\left(\partial_{\zeta}^{\alpha} b\right)^{\wedge}(\eta-\varsigma-\xi, \varsigma+t \xi) d t\right\} \times \xi^{\alpha} \hat{a}(\xi, \zeta) \hat{u}(\zeta) d \xi d \zeta
$$

(if $k=0$ the term in braces is replaced by $\hat{b}(\eta-\varsigma-\xi, \varsigma+\xi)$ ). Therefore,

$$
(r u)^{-}(\eta)=\int \hat{r}(\eta-\varsigma, \varsigma) \hat{u}(\varsigma) d \varsigma
$$


where

$$
\hat{r}(\eta, \zeta) \equiv \begin{cases}\iint \sum_{|\alpha|=k} c_{\alpha}\left\{\int_{0}^{1}(1-t)^{k-1}\left(\partial_{\zeta}^{\alpha} b\right)^{-}(\eta-\xi, \zeta+t \xi) d t\right\}\left(D_{x}^{\alpha} a\right)^{-}(\xi, \zeta) d \xi \\ \int \hat{b}(\eta-\xi, \zeta+\xi) \hat{a}(\xi, \zeta) d \xi & \text { if } k=0 .\end{cases}
$$

Let $\tilde{K} \subset \subset K$ be small conic neighborhoods of $\xi_{0}$, where $\left(x_{0}, \xi_{0}\right) \in \gamma$, with $K$ small enough that the estimates in Definition 1.2 hold for $\partial_{\xi}^{\alpha} b$ and $a$ on $K$. Set $\chi=\chi_{\tilde{K}}, \chi_{1}=\chi_{K}, \chi_{2}=\chi_{K^{c}}$. We must show that

$$
\frac{\langle\eta\rangle^{s} \hat{r}(\eta, \varsigma)}{\langle\varsigma\rangle^{m}} \in L^{2}(d \eta), \quad \frac{\langle\eta\rangle^{r} \chi_{\tilde{K}}(\eta) \hat{r}(\eta, \zeta)}{\langle\zeta\rangle^{m}} \in L^{2}(d \eta) \quad \text { uniformly in } \zeta .
$$

By (1.1) and the assumption on $\partial_{\xi}^{\alpha} b$ for $|\alpha|=k$, the integral kernels in the definition of $\hat{r}(\eta, \zeta)$ can be written

$$
\frac{\chi_{1}(\eta-\xi) g_{1}(\eta-\xi, \xi, \zeta)}{\langle\eta-\xi\rangle^{r}}+\frac{\chi_{2}(\eta-\xi) g_{2}(\eta-\xi, \xi, \zeta)}{\langle\eta-\xi\rangle^{s}}
$$

where $g_{i}(\eta, \xi, \zeta) \in L^{2}(d \eta)$ uniformly in $\xi, \varsigma$. And, by (1.1) and the assumption on $D_{x}^{\alpha} a$ for $|\alpha|=k$,

$$
\left(D_{x}^{\alpha} a\right)^{\wedge}(\xi, \zeta)=\frac{\chi_{1}(\xi) h_{1}(\xi, \zeta)\langle\zeta\rangle^{m}}{\langle\xi\rangle^{r}}+\frac{\chi_{2}(\xi) h_{2}(\xi, \zeta)\langle\zeta\rangle^{m}}{\langle\xi\rangle^{s}}
$$

where $h_{i}(\xi, \zeta) \in L^{2}(d \xi)$ uniformly in $\zeta$. Now (1.7) and the same argument as in the proof of Theorem 1.3, this time with $\zeta$ as a parameter, yield (1.8).

COROLlARY 1.6. Let $n / 2<s \leq r<2 s-n / 2$, let $k \geq 0$ be an integer, and assume that $a(x, \xi) \in S^{m+k ; s+k, r+k}(\gamma)$ and $\partial_{\xi}^{\alpha} b(x, \xi) \in S^{-|\alpha| ; s, r}(\gamma)$ for $|\alpha| \leq k$. Then $b(x, D) a(x, D)$ is an operator with symbol $b \circ a(x, \xi) \in S^{m+k ; s, r}(\gamma)$ satisfying

$$
\left\{b \circ a(x, \xi)-\sum_{|\alpha|<k} \frac{1}{\alpha !} \partial_{\xi}^{\alpha} b D_{x}^{\alpha} a(x, \xi)\right\} \in S^{m ; s, r}(\gamma) .
$$

ProOF. Let $\left\{\chi_{i}(\xi)\right\}$ be a smooth partition of unity on $\mathbf{R}^{n}$ with $\chi_{0}$ supported near zero, $\chi_{i}$ supported on $|\xi| \geq \frac{1}{2}$ and homogeneous of degree 0 on $|\xi| \geq 1$ for $i \neq 0$. Then $a(x, D)=\sum a(x, D) \chi_{i}(D)$. The term $a(x, D) \chi_{0}(D)$ is handled easily, so it can be assumed without loss of generality that $\left|\xi_{1}\right| \geq \frac{1}{2}$ on suppa. Let $D_{1}=(1 / i) \partial / \partial x_{1}$. By Leibnitz's rule,

$$
D_{1}^{k} \circ a(x, D)=a(x, D) D_{1}^{k}+\sum_{j=1}^{k} c_{j, k} D_{1}^{k-j} \circ\left(D_{1}^{j} a\right)(x, D)
$$

and we have

$$
\begin{aligned}
b(x, D) a(x, D)= & b(x, D) D_{1}^{k} \circ a(x, D) D_{1}^{-k} \\
& -\sum_{j=1}^{k} c_{j, k} b(x, D) D_{1}^{k-j} \circ\left(D_{1}^{j} a\right)(x, D) D_{1}^{-k} .
\end{aligned}
$$

Since $\partial_{\xi}^{\alpha}\left(b(x, \xi) \xi_{1}^{k-j}\right) \in S^{k-j-|\alpha| ; s, r}(\gamma)$ for $|\alpha| \leq k-j$ and $\left(D_{1}^{j} a\right)(x, \xi) \xi_{1}^{-k} \in$ $S^{m ; s+k-j, r+k-j}(\gamma)$, the result follows immediately from Lemma 1.5. 
LEMMA 1.7. Let $n / 2<s \leq r<2 s-n / 2, \mu \geq 0$, and let $k \geq 0$ be an integer. Assume that $a(x, \xi) \in S^{m ; s+k, r+k}(\gamma)$ and $b(x, \xi) \in S_{1,0}^{k+\mu}$. If $b \circ a(x, \xi)$ is the symbol of the composed operator $b(x, D) a(x, D)$, then there are symbols $r(x, \xi)$, $\tilde{r}(x, \xi) \in S^{m ; s, r}(\gamma)$ and $p(x, \xi), \tilde{p}(x, \xi) \in S_{1,0}^{\mu}$, such that

$$
\left\{b \circ a(x, D)-\sum_{|\alpha|<k} \frac{1}{\alpha !} \partial_{\xi}^{\alpha} b D_{x}^{\alpha} a(x, D)\right\}=r(x, D) p(x, D)+\tilde{p}(x, D) \tilde{r}(x, D) .
$$

PROOF. Let $r_{m+\mu}$ be the symbol of $b \circ a(x, D)-\sum_{|\alpha|<k}(1 / \alpha !) \partial_{\xi}^{\alpha} b D_{x}^{\alpha} a(x, D)$. Then from (1.7),

$$
\hat{r}_{m+\mu}(\eta, \xi)=\int \sum_{|\alpha|<k} c_{\alpha}\left\{\int_{0}^{1}(1-t)^{k-1}\left(\partial_{\zeta}^{\alpha} b\right)^{\wedge}(\eta-\xi, \zeta+t \xi) d t\right\}\left(D_{x}^{\alpha} a\right) \uparrow(\xi, \zeta) d \xi
$$

with the usual modification if $k=0$. Since $\left(\partial_{\zeta}^{\alpha} b\right)^{-}(\eta-\xi, \zeta+t \xi)$ is rapidly decreasing in $\eta-\xi$, it is easily seen that it suffices to consider the case $b(x, \xi)=b(\xi)$. Then

$$
\left(r_{m+\mu} u(\eta)\right)^{-}=\int \hat{r}_{m+\mu}(\eta-\xi, \xi) \hat{u}(\xi) d \xi
$$

where

$$
\hat{r}_{m+\mu}(\eta, \xi)=\left\{\begin{array}{l}
\sum_{|\alpha|=k} c_{\alpha} \int_{0}^{1} \partial_{\xi}^{\alpha} b(\xi+t \eta)\left(D_{x}^{\alpha} a\right)^{\wedge}(\eta, \xi) d t \quad \text { if } k \neq 0, \\
\partial_{\xi}^{\alpha} b(\xi+\eta) \hat{a}(\eta, \xi) \quad \text { if } k=0 .
\end{array}\right.
$$

By the assumption on $b$,

$$
\left|\partial_{\xi}^{\alpha} b(\xi+t \eta)\right| \leq c\left(\langle\xi\rangle^{\mu}+\langle\eta\rangle^{\mu}\right)
$$

for $|\alpha|=k, 0 \leq t \leq 1$. Define

$$
\begin{aligned}
& \hat{r}(\eta-\xi, \xi) \equiv \frac{\hat{r}_{m+\mu}(\eta-\xi, \xi) \chi(|\xi| \geq|\eta|)}{\langle\xi\rangle^{\mu}}, \\
& \hat{\tilde{r}}(\eta-\xi, \xi) \equiv \frac{\hat{r}_{m+\mu}(\eta-\xi, \xi) \chi(|\xi|<|\eta|)}{\langle\eta\rangle^{\mu}} .
\end{aligned}
$$

Then (1.9) implies that

$$
\begin{aligned}
& \left(r_{m+\mu} u\right)^{\wedge}(\eta)=\int \hat{r}(\eta-\xi, \xi)\langle\xi\rangle^{\mu} \hat{u}(\xi) d \xi+\langle\eta\rangle^{\mu} \int \hat{\tilde{r}}(\eta-\xi, \xi) \hat{u}(\xi) d \xi
\end{aligned}
$$

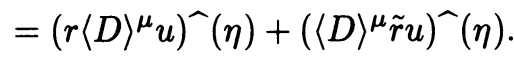

From (1.9) and (1.10) it follows that

$$
|\hat{r}(\eta, \xi)| \leq C \sum_{|\alpha|=k}\left|\left(D_{x}^{\alpha} a\right)^{\wedge}(\eta, \xi)\right| \quad \text { and } \quad|\hat{\tilde{r}}(\eta, \xi)| \leq C \sum_{|\alpha|=k}\left|\left(D_{x}^{\alpha} a\right)^{\uparrow}(\eta, \xi)\right|
$$

so by the assumption on $a, r(x, \xi)$ and $\tilde{r}(x, \xi)$ are symbols in $S^{m ; s, r}(\gamma)$. Finally,

$$
r_{m+\mu}(x, D)=r(x, D)\langle D\rangle^{\mu}+\langle D\rangle^{\mu} \tilde{r}(x, D)
$$

as desired. 
COROLlaRY 1.8. Let $n / 2<s \leq r<2 s-n / 2, \mu \geq 0$, and let $k>0$ be an integer. Assume that $a(x, \xi) \in S^{m+k ; s+k, r+k}(\gamma)$ and $b(x, \xi) \in S_{1,0}^{\mu}$. Then the conclusions of Lemma 1.7 hold.

Proof. As in the proof of Corollary 1.6 it suffices to assume that $\left|\xi_{1}\right| \geq 1 / 2$ on supp $a$ and write

$$
a(x, D)=D_{1}^{k} a(x, D) D_{1}^{-k}+\sum_{j=1}^{k} c_{j, k} D_{1}^{k-j}\left(D_{1}^{j} a\right)(x, D) D_{1}^{-k} .
$$

Since $b(x, \xi) \xi_{1}^{k-j} \in S_{1,0}^{\mu+k-j}$, and $D_{1}^{j} a(x, \xi) \xi_{1}^{-k} \in S^{m ; s+k-j, r+k-j}(\gamma)$, the result follows immediately.

The class of standard symbols, which includes all of those arising in the nonlinear problems to be considered below as well as appropriate expansions and remainders as given in the calculus lemmas above, may now be defined.

DEFINITION 1.9. For $k \geq 0$ an integer, $S_{s t}^{m+k ; s+k, r+k}(\gamma)$ consists of those symbols $a(x, \xi)$ of the form

$$
a_{m+k}(x, \xi)+a_{m+k-1}(x, \xi)+\cdots+a_{m}(x, \xi),
$$

where $a_{m}(x, \xi) \in S^{m ; s, r}(\gamma)$ and, for $0<j \leq k, a_{m+j}(x, \xi)=\sum a_{j, l}(x) p_{m+j, l}(x, \xi)$ with $a_{j, l}(x) \in H^{s+j} \cap H_{m l}^{s+j}(\gamma)$ and $p_{m+j, l}(x, \xi) \in S_{1,0}^{m+j}$.

One can easily see that

$$
\begin{aligned}
S_{s t}^{m+k ; s+k, r+k}(\gamma) \subset & S^{m+k ; s+k, r+k}(\gamma)+S^{m+k-1 ; s+k-1, r+k-1}(\gamma) \\
& +\cdots+S^{m ; s, r}(\gamma) \subset S^{m+k ; s, r}(\gamma)
\end{aligned}
$$

And, since

$$
\left\{H^{s+k} \cap H_{m l}^{r+k}(\gamma)\right\} \cdot\left\{H^{s+j} \cap H_{m l}^{r+j}(\gamma)\right\} \subset H^{s+j} \cap H_{m l}^{r+j}(\gamma)
$$

for $0 \leq j \leq k, n / 2<s<r<2 s-n / 2$, it follows that for such $s, r$, the product of an element of $S_{s t}^{m+k ; s+k, r+k}(\gamma)$ with an element of $S_{s t}^{\mu+k ; s+k, r+k}(\gamma)$ is in $S_{s t}^{m+\mu+2 k ; s+k, r+k}(\gamma)$. The useful features of the calculus of operators with these symbols are summarized below, extending the commutator lemma (corresponding to the case $k=1$ ) of $[\mathbf{1}]$.

THEOREM 1.10. Let $n / 2<s \leq r<2 s-n / 2$ and let $k \geq 0$ be an integer. Assume that $a(x, \xi) \in S_{s t}^{m+k ; s+k, r+k}(\gamma)$.

(i) If $\partial_{\xi}^{\alpha} b(x, \xi) \in S_{s t}^{-|\alpha| ; s+k, r+k}(\gamma)$ for $|\alpha| \leq k$, then $b(x, D) a(x, D)$ is an operator with symbol bo $a(x, \xi) \in S_{s t}^{m+k ; s+k, r+k}(\gamma)$ satisfying

$$
\left\{b \circ a(x, \xi)-\sum_{\substack{0 \leq j \leq k \\|\alpha|<j}} \frac{1}{\alpha !} \partial_{\xi}^{\alpha} b D_{x}^{\alpha} a_{m+j}(x, \xi)\right\} \in S^{m ; s, r}(\gamma) .
$$

(ii) If $b(x, \xi) \in S_{1,0}^{\mu+j}, \mu \geq 0,0 \leq j \leq k$, then

$$
\left\{b \circ a-\sum_{\substack{0 \leq i \leq k \\|\alpha|<i}} \frac{1}{\alpha !} \partial_{\xi}^{\alpha} b D_{x}^{\alpha} a_{m+i}\right\}(x, D)=r(x, D) p(x, D)+\tilde{p}(x, D) \tilde{r}(x, D)
$$

where $r, \tilde{r} \in S^{m+j ; s, r}(\gamma)$ and $p, \tilde{p} \in S_{1,0}^{\mu}$. 
(iii) If $m=0$ and $k \geq 1$, then the adjoint of $a_{k}$ is an operator with symbol $a_{k}^{*}(x, \xi) \in S_{s t}^{k ; s+k, r+k}(\gamma)$, and

$$
\left\{a_{k}^{*}(x, \xi)-\overline{a_{k}}(x, \xi)\right\} \in S_{s t}^{m+k-1 ; s+k-1, r+k-1}(\gamma) .
$$

PROOF. (i) and (ii) follow immediately from Corollaries 1.6 and 1.8, Lemma 1.7 and the algebraic properties of the symbols noted in the paragraph above. For (iii), since $k \geq 1, a_{k}=\sum a_{k, l}(x) p_{k, l}(x, \xi)$ so $a_{k}^{*}(x, D)=\sum p_{k, l}^{*}(x, D) \bar{a}_{k, l}(x)$, with $p_{k, l}^{*}(x, \xi) \in S_{1,0}^{k}$. The result now follows from the calculus of smooth pseudodifferential operators and Lemma 1.5.

In applications, the case $k=1$ is used when only the principal symbol of the composition of two operators is of concern, for instance as in [1] or when inverting in microlocally elliptic directions. But in general hyperbolic problems, the principal symbol of the commutator of certain operators must be kept under control (see Hörmander [8], Nirenberg [9]). Thus the terms of next lower order in the expansion of the compostions must be retained, corresponding to the case $k=2$ above.

The assumptions in Lemmas 1.5 and 1.7 are optimal in the sense that, if $k$ terms in the expansion for the composition are kept and the remainder is to be bounded on the appropriate $H^{s} \cap H_{m l}^{r}(\gamma)$ space, then the coefficients must be smoother of order $k$. For example, take $m=0, k=1, \mu=0$ in Lemma 1.5 or 1.7. Let $a(x, \xi)=a(x) \in H^{s+j} \cap H_{m l}^{r+j}(\gamma)$ and take $b(D)=D$, a first order differential operator. Then $D \circ a(x)-a(x) D=(D a)(x)$, and this operator is, in general, bounded on $H^{s} \cap H_{m l}^{r}(\gamma)$ only if $j \geq 1$ (and $n / 2<s \leq r<2 s-n / 2$ ). On the other hand, if $s$ is not too much larger than $n / 2$ and only the local spaces $H^{s}$ are involved, rather than the microlocal spaces, then the smoothness hypotheses for the analogue of Corollary 1.8 (and of Corollary 1.6, though this property will not be needed) may be relaxed. The conclusions will be weaker, implying for example that if $b_{0}(\xi) \in S_{1,0}^{0}, a(x) \in H^{s}$ and $p_{1}(\xi) \in S_{1,0}^{1}$, then $b_{0}(D) a(x) p_{1}(D)-a(x) b_{0}(D) p_{1}(D)$ has order less than one, rather than order zero as for classical pseudodifferential operators.

DEFINITION 1.11. Let $S^{m ; s}(U)$ for $U \subset \mathbf{R}^{n}$ denote the space of symbols as in Definition 1.2 without the microlocal hypotheses, that is,

$$
S^{m ; s}(U)=S^{m ; s, s}\left(U \times \mathbf{R}^{n} \backslash 0\right) .
$$

LEMMA 1.12. Let $n / 2<s \leq n / 2+1, \mu \geq 0$, and let $k \geq 1$ be an integer. Assume that $a(x, \xi) \in S^{m+k ; s+k-1}(U)$ and $b(x, \xi) \in S_{1,0}^{\mu}$. If $b \circ a(x, \xi)$ is the symbol of the composed operator $b(x, D) a(x, D)$, then for $0<\delta<s-n / 2$ there are symbols $r, \tilde{r} \in S^{m+1-\delta ; s-\delta}(U)$ and $p, \tilde{p} \in S_{1,0}^{\mu}$ satisfying

$$
\left\{b \circ a-\sum_{|\alpha|<k} \frac{1}{\alpha !} \partial_{\xi}^{\alpha} b D_{x}^{\alpha} a\right\}(x, D)=r(x, D) p(x, D)+\tilde{p}(x, D) r(x, D) .
$$

Moreover, if $\mu=0$ and $\delta \geq 1 / 2$, there are symbols $r_{0} \in S^{m+\delta ; s-\delta}(U)$ and $p_{0} \in$ $S_{1,0}^{1-2 \delta}$ such that the remainder above equals $p_{0}(x, D) r_{0}(x, D)$. In particular, for $m=\mu=0$ the remainder operator maps $H_{\mathrm{loc}}^{s}(U)$ to $H_{\mathrm{loc}}^{s-1+\delta}(U)$.

PROOF. In order to prove the last statement, notice that if $s \leq(n+1) / 2$, it follows that $s-\delta \geq s-1+\delta$, and since $r, \tilde{r} \in S^{1-\delta ; s-\delta}(U)$, by Theorem $1.3, r$ and 
$\tilde{r}$ map $H_{\mathrm{loc}}^{s}(U)$ to $H_{\mathrm{loc}}^{s-1+\delta}(U)$. On the other hand, $r_{0}: H_{\mathrm{loc}}^{s}(U) \rightarrow H_{\mathrm{loc}}^{s-\delta}(U)$ and $p_{0}(x, D): H_{\text {loc }}^{s-\delta}(U) \rightarrow H_{\text {loc }}^{s-1+\delta}(U)$, so the case $(n+1) / 2 \leq s \leq n / 2+1$ also follows.

To prove the main result, it suffices, as in the proof of Lemma 1.7, to assume that $b(x, \xi)=b(\xi)$. As in (1.9),

$$
\left\{\left(b \circ a-\sum_{|\alpha|<k} \frac{1}{\alpha !}\left(\partial_{\xi}^{\alpha} b\right)\left(\partial_{x}^{\alpha} a\right)\right) u\right\}^{\hat{}}(\eta)=\int \hat{r}_{m+\mu}(\eta-\xi, \xi) \hat{u}(\xi) d \xi
$$

where

$$
\hat{r}_{m+\mu}(\eta-\xi, \xi)=\sum_{|\alpha|=k} c_{\alpha} \int_{0}^{1}(1-t)^{k-1}\left(\partial_{\xi}^{\alpha} b\right)(\xi+t(\eta-\xi))(\eta-\xi)^{\alpha} \hat{a}(\eta-\xi, \xi) d t .
$$

And we may also write

$$
\hat{r}_{m+\mu}(\eta-\xi, \xi)=b(\eta) \hat{a}(\eta-\xi, \xi)-\sum_{|\alpha|<k} \frac{1}{\alpha !}\left(\partial_{\xi}^{\alpha} b\right)(\xi)(\eta-\xi)^{\alpha} \hat{a}(\eta-\xi, \xi) .
$$

Now define $r$ and $\tilde{r}$ by

$$
\begin{aligned}
\hat{r}(\eta-\xi, \xi) & \equiv \frac{\hat{r}_{m+\mu}(\eta-\xi, \xi) \chi(|\xi|>|\eta|)}{\langle\xi\rangle^{\mu}} \\
\hat{\tilde{r}}(\eta-\xi, \xi) & \equiv \frac{\hat{r}_{m+\mu}(\eta-\xi, \xi) \chi(|\xi| \leq|\eta|)}{\langle\eta\rangle^{\mu}}
\end{aligned}
$$

Then $\left(r_{m+\mu} u\right)^{\wedge}(\eta)=\left(r\langle D\rangle^{\mu} u\right)^{\wedge}(\eta)+\left(\langle D\rangle^{\mu} \tilde{r} u\right)^{\wedge}(\eta)$ so we need just check that $r$ and $\tilde{r}$ are in $S^{m+1-\delta ; s-\delta}(U)$. Setting $\tau=\eta-\xi$, it follows from (1.11) and (1.12), respectively, and the hypotheses on $a$ and $b$ that

$$
\begin{gathered}
|\hat{r}(\tau, \xi)| \leq \sup _{0 \leq t \leq 1} \frac{\langle\xi\rangle^{m+k}}{\langle\xi+t \tau\rangle^{k}\langle\tau\rangle^{s-1}} g_{1}(\tau, \xi), \\
|\hat{r}(\tau, \xi)| \leq \frac{\langle\xi\rangle^{m+k}}{\langle\tau\rangle^{s+k-1}}\left(1+\sum_{|\alpha|<k} \frac{\langle\tau\rangle|\alpha|}{\langle\xi\rangle|\alpha|}\right) g_{2}(\tau, \xi) \\
\leq C \frac{\langle\xi\rangle^{m+k}}{\langle\tau\rangle^{s+k-1}}\left(1+\frac{\langle\tau\rangle^{k-1}}{\langle\xi\rangle^{k-1}}\right) g_{2}(\tau, \xi)
\end{gathered}
$$

where $g_{i} \in L^{2}(d \tau)$ uniformly in $\xi$. Consequently,

$$
\left|\hat{r}(\tau, \xi) /\langle\xi\rangle^{m+1-\delta}\right| \leq M(\tau, \xi) g(\tau, \xi),
$$

where $g \in L^{2}(d \tau)$ uniformly in $\xi$, and

$$
M(\tau, \xi) \leq \frac{\langle\xi\rangle^{k-1+\delta}}{\langle\tau\rangle^{s-1}} \min \left(\sup _{0 \leq t \leq 1} \frac{1}{\langle\xi+t \tau\rangle^{k}}, \frac{1}{\langle\tau\rangle^{k}}+\frac{1}{\langle\tau\rangle\langle\xi\rangle^{k-1}}\right) .
$$

If $|\tau|>\frac{1}{2}|\xi|$, then

$$
\left(1 /\langle\tau\rangle^{k}+1 /\langle\tau\rangle\langle\xi\rangle^{k-1}\right) \leq C /\langle\tau\rangle\langle\xi\rangle^{k-1}
$$


so $M \leq\langle\xi\rangle^{\delta} /\langle\tau\rangle^{s} \leq C /\langle\tau\rangle^{s-\delta}$. And if $|\tau| \leq \frac{1}{2}|\xi|, \xi+t \tau \approx \xi$ for $0 \leq t \leq 1$, so

$$
M \leq C /\langle\xi\rangle^{1-\delta}\langle\tau\rangle^{s-1} \leq C /\langle\tau\rangle^{s-\delta}, \quad \text { since } \delta \leq 1 .
$$

Thus $r \in S^{m+1-\delta ; s-\delta}(U)$. A similar proof shows that $\tilde{r} \in S^{m+1-\delta ; s-\delta}(U)$.

Finally, suppose that $\mu=0$ and $\delta \geq \frac{1}{2}$. If we define

$$
\hat{r}_{0}(\eta-\xi, \xi) \equiv \hat{r}_{m+\mu}(\eta-\xi, \xi) /\langle\eta\rangle^{1-2 \delta}
$$

then

$$
\left(r_{m+\mu} u\right)^{\uparrow}(\eta)=\left(\langle D\rangle^{1-2 \delta} r_{0} u\right)^{\uparrow}(\eta),
$$

so we need just check that $r_{0} \in S^{m+\delta ; s-\delta}(U)$. This is proven, as above, by using (1.11) and (1.12) and the properties of $b$ and $a$ to show that $\langle\tau\rangle^{s-\delta}\left|\hat{r}_{0}(\tau, \xi)\right|\langle\xi\rangle^{-(m+\delta)}$ - $\quad \leq C g(\tau, \xi)$ where $g \in L^{2}(d \tau)$ uniformly in $\xi$.

2. Linear and nonlinear elliptic theorems. The calculus developed in $\S 1$ allows an easy proof of the following microlocal elliptic regularity theorem.

THEOREM 2.1. Let $n / 2<s \leq r<2 s-n / 2$. Assume that

$$
a(x, \xi) \in S_{s t}^{m+1 ; s+1, r+1} \text {. }
$$

\section{Suppose}

(i) $v \in H^{s+m} \cap H_{m l}^{r+m}\left(p_{0}\right)$,

(ii) $f \in H^{s} \cap H_{m l}^{r}\left(p_{0}\right)$,

(iii) $a(x, D) v=f$.

Then, $v \in H^{s+m} \cap H_{m l}^{r+m+1}\left(p_{0}\right)$.

ProOF. Since $a \in S_{s t}^{m+1 ; s+1, r+1}$ at $p_{0}$ we may write $a=a_{m+1}+a_{m}$ where $a_{m+l} \in S^{m+l ; s+l, r+l}$. Let $b_{0} \in S_{1,0}^{0}$ have support near enough to $p_{0}$ so that $a_{m+1}$ is elliptic on $\operatorname{supp} b_{0}$. Set

$$
c_{0}=b_{0}(x, \xi)\langle\xi\rangle^{m+1} / a_{m+1}(x, \xi) .
$$

Since $H^{s+1} \cap H_{m l}^{r+1}\left(p_{0}\right)$ is an algebra, it follows easily that

$$
\partial_{\xi}^{\alpha} c_{0}(x, \xi) \in S^{-|\alpha| ; s+1, r+1}\left(p_{0}\right) \text { for all } \alpha .
$$

By Theorem 1.10(i), there is a symbol $r(x, \xi) \in S_{s t}^{m ; s, r}\left(p_{0}\right)$ such that

$$
c_{0}(x, D) a(x, D)=b_{0}(x, D)\langle D\rangle^{m+1}+r(x, D) .
$$

Thus (iii) implies

$$
b_{0}(x, D)\langle D\rangle^{m+1} v=c_{0}(x, D) f-r(x, D) v .
$$

Theorem 1.3 implies that $c_{0}(x, D) f \in H^{s} \cap H_{m l}^{r}$ and $r(x, D) v \in H^{s} \cap H_{m l}^{r}$. Therefore (2.1) and the standard microlocal elliptic result imply that $v \in H^{s+m} \cap$ $H_{m l}^{r+m+1}\left(p_{0}\right)$.

We now present several local elliptic theorems, both linear and nonlinear, which follow easily from Theorem 2.1. The full (microlocal) strength of Theorem 2.1 will be used in the proof of the propagation theorem in $\S 3$ and the examples in $\S 4$. 
THEOREM 2.2. Let $L=\sum_{0 \leq|\alpha| \leq 2 l} b_{\alpha}(x) D^{\alpha}$ for some integer $l>0$ and suppose that $L$ is elliptic at $x_{0}$, i.e. $\sum_{|\alpha|=2 l} b_{\alpha}\left(x_{0}\right) \xi^{\alpha} \geq c|\xi|^{2 l}$. Suppose that $s>n / 2+2 l$ and

(i) $u \in H^{s}\left(x_{0}\right)$,

(ii) $f \in H^{s}\left(x_{0}\right), b_{\alpha} \in H^{s}\left(x_{0}\right)$ for all $\alpha$,

(iii) $L u=f$.

Then $u \in H^{s+2 l}\left(x_{0}\right)$.

PROOF. Let $\xi_{0}$ be given and let $\partial$ be a directional derivative for which $\xi_{0}$ is noncharacteristic. Applying $\partial$ to both sides of (iii), we have

$$
\begin{aligned}
\sum_{|\alpha|=2 l} b_{\alpha}(x) D^{\alpha} \partial u=\partial f & -\sum_{|\alpha|<2 l} b_{\alpha}(x) D^{\alpha} \partial u \\
& -\sum_{0 \leq|\alpha| \leq 2 l}\left(\partial b_{\alpha}\right)(x) D^{\alpha} u \in H^{s-2 l}\left(x_{0}\right) .
\end{aligned}
$$

Setting $v=(1-\Delta)^{l} u$ and $A(x, D)=\sum_{|\alpha|=2 l} b_{\alpha}(x) D^{\alpha} \partial(1-\Delta)^{-l}$ we find that $A(x, D) v \in H^{s-2 l}\left(x_{0}\right)$ and $A(x, \xi) \in S^{0+1 ; s+1}\left(x_{0}\right)$ since $b_{\alpha} \in H^{s} \supset H^{s-2 l+1}$. Furthermore, $A(x, \xi)$ is elliptic at $\left(x_{0}, \xi_{0}\right)$; so by Theorem 2.1,

$$
v \in H^{s-2 l} \cap H_{m l}^{s-2 l+1}\left(x_{0}, \xi_{0}\right)
$$

which implies that

$$
u \in H^{s} \cap H_{m l}^{s+1}\left(x_{0}, \xi_{0}\right) .
$$

Since $\xi_{0}$ was arbitrary, we have $u \in H^{s+1}\left(x_{0}\right)$. Repeating this procedure yields $u \in H^{s+2 l}\left(x_{0}\right)$.

A quasilinear analogue of Weyl's lemma follows immediately from Theorem 2.2.

THEOREM 2.3. Assume $|\beta| \leq 2 l-1$ and suppose that $u \in H^{s}\left(x_{0}\right), s>n / 2+$ $2 l+|\beta|$, is a solution of

$$
\sum_{|\alpha|=2 l} b_{\alpha}\left(x, u, \ldots, D^{\beta} u\right) D^{\alpha} u=f\left(x, u, \ldots, D^{\beta} u\right)
$$

in a neighborhood of $x_{0}$. If $b_{\alpha}, f \in C^{\infty}$ and if $L=\sum_{|\alpha|=2 l} b_{\alpha}\left(x, u, \ldots, D^{\beta} u\right) D^{\alpha}$ is elliptic at $x_{0}$ in the sense that

$$
\sum_{|\alpha|=2 l} b_{\alpha}\left(x_{0}, u\left(x_{0}\right), \ldots, D^{\beta} u\left(x_{0}\right)\right) \xi^{\alpha} \geq c(1+|\xi|)^{2 l} .
$$

for $\xi$ large, then $u \in C^{\infty}$ near $x_{0}$.

PROOF. Simply apply Theorem 2.2 repeatedly.

3. Propagation of singularities. We shall prove first the analogue of the strong Gårding inequality for symbols in $S^{m ; s}(U)$. The proof is particularly simple because very few conditions need to be checked to verify that remainders are in $S^{0 ; s}$. 
LEMMA 3.1. Let $n / 2<s, 0<\varepsilon \leq 1$, and suppose that $p(x, \xi) \in S^{\varepsilon ; s+2 \varepsilon}(U)$ and satisfies $p(x, \xi) \geq 0$. Then, for all $u \in H^{0}(U)$ with compact support in $U$,

$$
\operatorname{Re}\langle p(x, D) u, u\rangle \geq-C\|u\|_{H^{0}(U)}^{2} .
$$

Proof. As in Taylor [14, Chapter VII], let $b(D, x, D)$ be the Friedrichs symmetrization of $p$. Set $r(x, D)=b(D, x, D)-p(x, D)$. Suppose that we can prove that $r \in S^{0 ; s}(U)$. Then by Theorem 1.3, $r(x, D)$ is bounded on $H_{\text {loc }}^{0}$ so (3.1) would follow since $\langle b(D, x, D) u, u\rangle \geq 0$.

Let $q$ be a smooth nonnegative even function, supported in $|\xi| \leq 1$, satisfying $\int q^{2}(\xi) d \xi=1$, and define

$$
\begin{gathered}
F(\xi, \varsigma) \equiv \frac{1}{\langle\xi\rangle^{n / 4}} q\left(\frac{\varsigma-\xi}{\langle\xi\rangle^{1 / 2}}\right) \\
b(\eta, x, \xi) \equiv \int F(\eta, \varsigma) p(x, \varsigma) F(\xi, \varsigma) d \varsigma .
\end{gathered}
$$

Then,

$$
(b(D, x, D) u)^{\wedge}(\eta)=\int \hat{b}(\eta, \eta-\xi, \xi) \hat{u}(\xi) d \xi
$$

Thus,

$$
(r(x, D) u)^{\wedge}(\eta)=\int \hat{r}(\eta-\xi, \xi) \hat{u}(\xi) d \xi
$$

where

$$
\begin{aligned}
\hat{r}(\eta, \xi)= & \hat{b}(\eta+\xi, \eta, \xi)-\hat{p}(\eta, \xi) \\
= & \int F(\eta+\xi, \varsigma) \hat{p}(\eta, \varsigma) F(\xi, \varsigma) d \varsigma-\hat{p}(\eta, \xi) \\
= & \int F(\eta+\xi, \varsigma)\{\hat{p}(\eta, \varsigma)-\hat{p}(\eta, \xi)\} F(\xi, \varsigma) d \varsigma \\
& +\int\{F(\eta+\xi, \varsigma)-F(\xi, \varsigma)\} \hat{p}(\eta, \xi) F(\xi, \varsigma) d \xi
\end{aligned}
$$

since $\int F^{2}(\xi, \zeta) d \zeta=1$. We need to show that

$$
\langle\eta\rangle^{s}|\hat{r}(\eta, \xi)| \leq C g(\eta, \xi)
$$

where $g \in L^{2}(d \eta)$ uniformly in $\xi$. On supp $F(\xi, \zeta),|\zeta-\xi| \leq\langle\xi\rangle^{1 / 2}$, so for large $\xi$ we have $\zeta \approx \xi$. It follows from (3.2) and the assumptions on $p$ that

$$
|\hat{r}(\eta, \xi)| \leq \frac{\langle\xi\rangle^{\varepsilon}}{\langle\eta\rangle^{s+2 \varepsilon}} g(\eta, \xi)
$$

so (3.4) certainly holds for $|\eta| \geq \frac{1}{2}|\xi|$. From now on assume that $|\eta| \leq \frac{1}{2}|\xi|$. Then, for $\bar{\eta}$ on the segment between 0 and $\eta$ and for $(\xi, \eta) \in \operatorname{supp} F$, we have

$$
\varsigma \approx \xi \approx \bar{\eta}+\xi, \quad|\zeta-\xi| \leq\langle\xi\rangle^{1 / 2}, \quad|\varsigma-\xi| \leq c\langle\bar{\eta}+\xi\rangle^{1 / 2} .
$$

Now, for $\varsigma \approx \xi$,

$$
\hat{p}(\eta, \varsigma)-\hat{p}(\eta, \xi)=(\varsigma-\xi) \cdot \nabla_{\xi} p(\eta, \xi)+O\left(|\varsigma-\xi|^{2}\left|\partial_{\xi}^{2} \hat{p}(\eta, \xi)\right|\right)
$$


and, since $F$ is even,

$$
\begin{aligned}
\int F(\eta+\xi, \varsigma)\{\hat{p}(\eta, \varsigma)-\hat{p}(\eta, \xi)\} F(\xi, \zeta) d \zeta \\
=\nabla_{\xi} \hat{p}(\eta, \xi) \cdot \int(\varsigma-\xi)(F(\eta+\xi, \zeta)-F(\xi, \zeta)) d \zeta \\
\quad+O\left(\left|\partial_{\xi}^{2} p(\eta, \xi)\right|\right) \int|\varsigma-\xi|^{2}|F(\eta+\xi, \varsigma)-F(\xi, \zeta)| d \zeta
\end{aligned}
$$

It follows from (3.3) and the assumptions on $p$ that

$$
|\hat{r}(\eta, \xi)| \leq \tilde{M}(\eta, \xi) \tilde{g}(\eta, \xi)
$$

where $\tilde{g}(\eta, \xi) \in L^{2}(d \eta)$ uniformly in $\xi$, and

$$
\begin{aligned}
\tilde{M}(\eta, \xi)= & \frac{\langle\xi\rangle^{\varepsilon}}{\langle\eta\rangle^{s+2 \varepsilon}} \int\left(\frac{|\zeta-\xi|}{\langle\xi\rangle}+\frac{|\zeta-\xi|^{2}}{\langle\xi\rangle^{2}}\right)|F(\eta+\xi, \zeta)-F(\xi, \zeta)| F(\xi, \zeta) d \zeta \\
& +\frac{\langle\xi\rangle^{\varepsilon}}{\langle\eta\rangle^{s+2 \varepsilon}}\left|\int(F(\eta+\xi, \varsigma)-F(\xi, \zeta)) F(\xi, \zeta) d \zeta\right| .
\end{aligned}
$$

Let $M_{1}, M_{2}, M_{3}$ be the parts of $\tilde{M}$ corresponding to $|\zeta-\xi|\langle\xi\rangle^{-1},|\zeta-\xi|^{2}\langle\xi\rangle^{-2}$ and the second integral, respectively. $F$ satisfies

$$
\begin{aligned}
\nabla_{\xi} F & =\frac{a(\xi)}{\langle\xi\rangle^{n / 4}} q_{1}\left(\frac{\zeta-\xi}{\langle\xi\rangle^{1 / 2}}\right)+\frac{b(\xi)}{\langle\xi\rangle^{n / 4}} \nabla q\left(\frac{\varsigma-\xi}{\langle\xi\rangle^{1 / 2}}\right), \\
\partial_{\xi}^{2} F & =\left\{\frac{1}{\langle\xi\rangle^{n / 4}} q_{2}\left(\frac{\zeta-\xi}{\langle\xi\rangle^{1 / 2}}\right)\right\} O\left(\frac{1}{\langle\xi\rangle}\right)
\end{aligned}
$$

where $q_{i} \in C_{0}^{\infty}, a(\xi)=O\left(\langle\xi\rangle^{-1}\right), b(\xi)=O\left(\langle\xi\rangle^{-1 / 2}\right)$. Now, from (3.6), we have

$$
M_{2} \leq \frac{C\langle\xi\rangle^{\varepsilon}}{\langle\eta\rangle^{s+2 \varepsilon}\langle\xi\rangle} .
$$

Writing $F(\eta+\xi, \varsigma)-F(\xi, \varsigma)=\eta \cdot \nabla_{\xi} F(\bar{\eta}+\xi, \varsigma)$, and using (3.6), we have

$$
M_{1} \leq \frac{C\langle\xi\rangle^{\varepsilon}\langle\eta\rangle}{\langle\eta\rangle^{s+2 \varepsilon}\langle\xi\rangle} \text {. }
$$

And, because

$$
F(\eta+\xi, \varsigma)-F(\xi, \varsigma)=\eta \cdot \nabla_{\xi} F(\xi, \varsigma)+O\left(|\eta|^{2} \partial_{\xi}^{2} F(\xi+\bar{\eta}, \varsigma)\right)
$$

and

$$
\frac{b(\xi)}{\langle\xi\rangle^{n / 2}} \int \nabla q\left(\frac{\zeta-\xi}{\langle\xi\rangle^{1 / 2}}\right) q\left(\frac{\varsigma-\xi}{\langle\xi\rangle^{1 / 2}}\right) d \xi=0
$$

since $q$ is even, it follows from (3.6) that

$$
M_{3} \leq C\left(\langle\xi\rangle^{\varepsilon} /\langle\xi\rangle^{s+2 \varepsilon}\right)\left(\langle\eta\rangle+\langle\eta\rangle^{2} /\langle\xi\rangle\right) .
$$

Putting together these estimates on the $M_{i}$ with (3.5) shows that, for any $\alpha$ with $0 \leq \alpha \leq 1$

$$
|\hat{r}(\eta, \xi)| \leq \frac{\langle\xi\rangle^{\varepsilon}}{\langle\eta\rangle^{s+2 \varepsilon}}\left(\frac{\langle\eta\rangle^{2}}{\langle\xi\rangle}\right)^{\alpha} g_{\alpha}(\eta, \xi)
$$

for $|\eta| \leq \frac{1}{2}|\xi|$, for some $g_{\alpha} \in L^{2}(d \eta)$ uniformly in $\xi$. The choice $\alpha=\varepsilon$ yields (3.4) as desired. 
We now prove the main result on propagation of singularities for equations with nonsmooth coefficients. As in the smooth case, the key ingredient is the microlocal cutoff which reduces the microlocal problem to a local one. We note that if $a(x, \xi) \in$ $S_{s t}^{m+2 ; s+2}(U)$ has real principal symbol $a_{m+2}(x, \xi)$ homogeneous in $\xi$ of degree $m+2$ and $s>n / 2$, then by Sobolev's lemma, $\nabla_{x} a_{m+2} \in C^{1}\left(U \times \mathbf{R}^{n} \backslash\{0\}\right)$ and $\nabla_{\xi} a_{m+2} \in C^{2}\left(U \times \mathbf{R}^{n} \backslash\{0\}\right)$. Thus, if $U$ is a small neighborhood of the characteristic point $\left(x_{0}, \xi_{0}\right)$ of $a_{m+2}$, there is in $U \times \mathbf{R}^{n} \backslash\{0\}$ a unique $C^{2}$ null bicharacteristic $\gamma$ of $a_{m+2}$ through $\left(x_{0}, \xi_{0}\right)$ defined by the equations $\dot{x}=\nabla_{\xi} a_{m+2}, \dot{\xi}=-\nabla_{x} a_{m+2}$.

THEOREM 3.2. Let $n / 2<s \leq r<2 s-n / 2$. Assume that

$$
a(x, \xi) \in S_{s t}^{m+2 ; s+2, r+2}(\gamma)
$$

has real principal symbol $a_{m+2}$ which is homogeneous of degree $m+2$ in $\xi$, and that $\gamma$ is the null bicharacteristic through the characteristic point $\left(x_{0}, \xi_{0}\right)$. If:

(i) $v \in H^{s+m} \cap H_{m l}^{r+m}(\gamma)$,

(ii) $v \in H^{s+m} \cap H_{m l}^{r+m+\varepsilon}\left(x_{0}, \xi_{0}\right)$ for some $0<\varepsilon \leq 1$,

(iii) $f \in H^{s} \cap H_{m l}^{r}(\gamma)$,

(iv) $a(x, D) v=f$.

Then $v \in H^{s+m} \cap H_{m l}^{r+m+\varepsilon}(\gamma)$.

PROOF. We may assume that the assumptions hold with $\gamma$ and $\left(x_{0}, \xi_{0}\right)$ replaced with conic neighborhoods $\Gamma$ and $\Gamma_{0}$, respectively. By replacing $a(x, \xi)$ by $a(x, \xi)\langle\xi\rangle^{-m-1}$ and $v$ by $\langle D\rangle^{m+1} v$, we may assume that $m=-1$. Then $a(x, \xi)=a_{1}(x, \xi)+a_{0}(x, \xi)+a_{-1}(x, \xi)$ where $a_{j}$ has order $j$, and smoothness $H^{s+j+1} \cap H_{m l}^{r+j+1}(\gamma)$.

Let $e_{0}^{\delta}(\xi)=\left(1+\delta|\xi|^{2}\right)^{-1 / 2}$ for $0<\delta \leq 1$. For each $\delta, e_{0}^{\delta} \in S_{1,0}^{-1}$ and the collection $\left\{e_{0}^{\delta}\right\}$ is a bounded subset of $S_{1,0}^{0}$. Also, $D_{\xi} e_{0}^{\delta}=i \delta \xi\left(1+\delta|\xi|^{2}\right)^{-1} e_{0}^{\delta} \equiv r_{-1}^{\delta} e_{0}^{\delta}$, where $\left\{r_{-1}^{\delta}\right\}$ is a bounded subset of $S_{1,0}^{-1}$. By Theorem 1.10,

$$
\begin{aligned}
e_{0}^{\delta}(D) a(x, D)= & a_{1}(x, D) e_{0}^{\delta}(D)+\left(\nabla_{x} a_{1}(x, D) \cdot r_{-1}^{\delta}(D)\right) e_{0}^{\delta}(D) \\
& +a_{0}(x, D) e_{0}^{\delta}(D)+\tilde{a}_{-1, \delta}(x, D) \\
\equiv & a_{1}(x, D) e_{0}^{\delta}(D)+\tilde{a}_{0, \delta}(x, D) e_{0}^{\delta}(D)+\tilde{a}_{-1, \delta}(x, D)
\end{aligned}
$$

where $\left\{\tilde{a}_{0, \delta}\right\} \subset S_{s t}^{0 ; s+1, r+1}(\Gamma)$ and $\left\{\tilde{a}_{-1, \delta}\right\} \subset S^{-1 ; s, r}(\Gamma)$ are bounded subsets. Set $v_{\delta}=e_{0}^{\delta}(D) v$ and $f_{\delta}=-a_{-1, \delta}(x, D) v+e_{0}^{\delta}(D) f$. Then $v_{\delta} \in H^{s} \cap H_{m l}^{r}(\Gamma)$ (with norm depending on $\delta$ ) and by Theorem $1.3, f_{\delta} \in H^{s} \cap H_{m l}^{r}(\Gamma)$ with norm independent of $\delta$. The equation $\left(a_{1}(x, D)+\tilde{a}_{0, \delta}(x, D)\right) v_{\delta}=f_{\delta}$ holds and from the hypotheses it follows that $v_{\delta} \in H^{s-1} \cap H_{m l}^{r-1}(\Gamma)$ and $v_{\delta} \in H_{m l}^{r-1+\varepsilon}\left(\Gamma_{0}\right)$ with norms independent of $\delta$. Thus, it suffices to assume that $a(x, \xi) \in S_{s t}^{1 ; s+2, r+2}(\Gamma)$ and

(i) $v \in H^{s-1} \cap H_{m l}^{r-1}(\Gamma)$

(ii) $v \in H_{m l}^{r-1+\varepsilon}\left(\Gamma_{0}\right)$ for some $0 \leq \varepsilon \leq 1$,

(iii) $f \in H^{s} \cap H_{m l}^{r}(\Gamma)$,

(iv) $a(x, D) v=f$,

and that $\|v\|_{H_{m l}^{r-1+\varepsilon}(\gamma)}$ is finite, and to prove that $\|v\|_{H_{m l}^{r-1+\varepsilon}(\gamma)}$ depends only on the norms of $a, v, f$ in (i), (ii) and (iii). Finally, by induction it suffices to replace (i) and (ii) by

(i) $v \in H^{s-1} \cap H^{\tilde{r}-\alpha / 2}(\Gamma)$, 
(ii) $v \in H_{m l}^{\tilde{r}}\left(\Gamma_{0}\right)$,

and to estimate $\|v\|_{H_{m l}^{r}(\gamma)}$. Here $\alpha>0, r-1+\alpha / 2 \leq \tilde{r} \leq r-1+\varepsilon$, and we can assume that $\alpha \leq 1$.

If the Hamiltonian vector field $H_{a_{1}}$ is zero at $\left(x_{0}, \xi_{0}\right), \gamma$ is the point $\left(x_{0}, \xi_{0}\right)$, while if $H_{a_{1}}$ has the radial cotangent direction, $\gamma$ is the ray $\left(x_{0}, r \xi_{0}\right), r>0$. In either case nothing needs to be proven, so we assume that $H_{a_{1}}$ is neither zero nor radial.

Following Bony $[4, \S 6]$, we can find (since $a_{1} \in C^{2}$ ) a $b_{\tilde{r}}(x, \xi) \in S_{1,0}^{\tilde{r}}$ which is real, homogeneous of degree $\tilde{r}$, with support in $\Gamma$, such that $H_{a_{1}} b_{\tilde{r}}^{2} \geq 0$ on $\Gamma \backslash \Gamma_{0}$ and $H_{a_{1}} b_{\tilde{r}}^{2}>0$ on $\gamma \backslash \Gamma_{0}$, and a $b_{0} \in S_{1,0}^{0}$ with $H_{a_{1}} b_{0} \geq 1$ on $\Gamma$. Let $b_{\tilde{r}}^{(M)}=b_{\tilde{r}} e^{M b_{0}}$. Then

$$
\begin{aligned}
\frac{1}{2} H_{a_{1}}\left(b_{\tilde{r}}^{(M)}\right)^{2} & =M\left(H_{a_{1}} b_{0}\right)\left(b_{\tilde{r}}^{2} e^{2 M b_{0}}\right)+\frac{1}{2}\left(H_{a_{1}} b_{\tilde{r}}^{2}\right) e^{2 M b_{0}} \\
& \geq M\left(b_{\tilde{r}}^{(M)}\right)^{2}+c\langle\xi\rangle^{2 \tilde{r}} \text { on } \gamma \backslash \Gamma_{0} \\
& \geq M\left(b_{\tilde{r}}^{(M)}\right)^{2} \quad \text { on } \Gamma \backslash \Gamma_{0} .
\end{aligned}
$$

It follows that there are symbols $b_{2 \tilde{r}}, e_{2 \tilde{r}} \in S_{1,0}^{2 \tilde{r}}$ with $b_{2 \tilde{r}}$ supported on $\Gamma_{0}, e_{2 \tilde{r}}$ supported on $\Gamma$ and elliptic on $\gamma$, such that

$$
\frac{1}{2} H_{a_{1}}\left(b_{\tilde{r}}^{(M)}\right)^{2}-M\left(b_{\tilde{r}}^{(M)}\right)^{2}+b_{2 \tilde{r}} \geq e_{2 \tilde{r}}
$$

Note that the principal symbol of $(1 / i) b_{\tilde{r}}^{(M)}(x, D)^{*}\left[b_{\tilde{r}}^{(M)}(x, D), a_{1}(x, D)\right]$ is

$$
\frac{1}{2} H_{a_{1}}\left(b_{\tilde{r}}^{(M)}\right)^{2}(x, \xi) \in S_{s t}^{2 \tilde{r} ; s+1, r+1}(\Gamma) .
$$

Thus,

$$
\begin{aligned}
\operatorname{Re} \frac{1}{i}\left\langle b_{\tilde{r}}^{(M)^{*}}\left[b_{\tilde{r}}^{(M)}, a_{1}\right] v, v\right\rangle= & \operatorname{Im}\left(\left\langle b_{\tilde{r}}^{(M)} a_{1} v, b_{\tilde{r}}^{(M)} v\right\rangle-\left\langle a_{1} b_{\tilde{r}}^{(M)} v, b_{\tilde{r}}^{(M)} v\right\rangle\right) \\
= & \operatorname{Im}\left\langle b_{\tilde{r}}^{(M)} a_{1} v, b_{\tilde{r}}^{(M)} v\right\rangle-\frac{1}{2 i}\left\langle\left(a_{1}-a_{1}^{*}\right) b_{\tilde{r}}^{(M)} v, b_{\tilde{r}}^{(M)} v\right\rangle \\
= & \operatorname{Im}\left\langle-b_{\tilde{r}}^{(M)} a_{0} v, b_{\tilde{r}}^{(M)} v\right\rangle+\operatorname{Im}\left\langle b_{\tilde{r}}^{(M)} f, b_{\tilde{r}}^{(M)} v\right\rangle \\
& -\frac{1}{2 i}\left\langle\left(a_{1}-a_{1}^{*}\right) b_{\tilde{r}}^{(M)} v, b_{\tilde{r}}^{(M)} v\right\rangle .
\end{aligned}
$$

Since $a_{1}(x, \xi)$ is real, Theorem 1.10 (iii) with $m=0$ and $k=1$ implies $\left(a_{1}-a_{1}^{*}\right) \in$ $S^{0 ; s, r}(\Gamma)$. (Note that here we have only used the fact that $a_{1} \in S_{s t}^{1 ; s+1, r+1}(\Gamma)$ rather than $S_{s t}^{1 ; s+2, r+2}(\Gamma)$.) Therefore

$$
\left|\frac{1}{2 i}\left\langle\left(a_{1}-a_{1}^{*}\right) b_{\tilde{r}}^{(M)} v, b_{\tilde{r}}^{(M)} v\right\rangle\right| \leq C_{0}\left\|b_{\tilde{r}}^{(M)} v\right\|_{H^{0}}^{2}
$$

with $C_{0}$ independent of $M$, by Theorem 1.3 and the hypothesis on $a$. Further,

$$
\left|\operatorname{Im}\left\langle b_{\tilde{r}}^{(M)} f, b_{\tilde{r}}^{(M)} v\right\rangle\right| \leq C_{M}+\left\|b_{\tilde{r}}^{(M)} v\right\|_{H^{0}}^{2}
$$

by (iii). Finally,

$$
\left\langle b_{\tilde{r}}^{(M)} a_{0} v, b_{\tilde{r}}^{(M)} v\right\rangle=\left\langle a_{0} b_{\tilde{r}}^{(M)} v, b_{\tilde{r}}^{(M)} v\right\rangle+\left\langle\left[b_{\tilde{r}}^{(M)}, a_{0}\right] v, b_{\tilde{r}}^{(M)} v\right\rangle .
$$

For the first term on the right,

$$
\left|\left\langle a_{0} b_{\tilde{r}}^{(M)} v, b_{\tilde{r}}^{(M)} v\right\rangle\right| \leq C_{1}\left\|b_{\tilde{r}}^{(M)} v\right\|_{H^{0}}^{2}
$$


by Theorem 1.3 since $a_{0} \in S^{0 ; s+1, r+1}$. By Theorem 1.10(ii) with $\mu=\tilde{r}-1$, $j=1, k=1, m=-1$, we have $\left[b_{\tilde{r}}^{(M)}, a_{0}\right]=a_{0} p^{(M)}+\tilde{p}^{(M)} \tilde{a}_{0}$, where $a_{0}, \tilde{a}_{0} \in$ $S^{0 ; s, r}, p^{(M)}, \tilde{p}^{(M)} \in S_{1,0}^{\tilde{r}-1}$. Since $\operatorname{supp} b_{\tilde{r}}^{(M)} \subset \Gamma$, for appropriate $b_{0}^{(M)} \in S_{1,0}^{0}$ with $\operatorname{supp} b_{0}^{(M)} \subset \Gamma, b_{0}^{*}(x, D) b_{\tilde{r}}^{(M)}(x, D)=b_{\tilde{r}}^{(M)}(x, D)$ so

$$
\left\langle\left[b_{\tilde{r}}^{(M)}, a_{0}\right] v, b_{\tilde{r}}^{(M)} v\right\rangle=\left\langle b_{0}^{(M)}\left(a_{0} p^{(M)}+\tilde{p}^{(M)} a_{0}\right) v, b_{\tilde{r}}^{(M)} v\right\rangle .
$$

Since $a_{0} p^{(M)} \in S^{\tilde{r}-1 ; s, r}(\Gamma)$, Theorem 1.3 and (i) imply $\left\|b_{0}^{(M)} a_{0} p^{(M)} v\right\|_{H^{1-\alpha / 2}} \leq$ $C_{M}$, and since $\tilde{a}_{0}: H_{m l}^{s-1, \tilde{r}-\alpha / 2}(\Gamma) \rightarrow H_{m l}^{s-1, \tilde{r}-\alpha / 2}(\Gamma)$, we have

$$
\left\|b_{0}^{(M)} \tilde{p}^{(M)} \tilde{a}_{0} v\right\|_{H^{1-\alpha / 2}} \leq C_{M} .
$$

Thus,

$$
\left|\left\langle\left[b_{\tilde{r}}^{(M)}, a_{0}\right] v, b_{\tilde{r}}^{(M)} v\right\rangle\right| \leq C_{M}+\left\|b_{\tilde{r}}^{(M)} v\right\|_{H^{0}}^{2} .
$$

Together the above estimates yield

$$
\operatorname{Re} \frac{1}{i}\left\langle b_{\tilde{r}}^{(M)^{*}}\left[b_{\tilde{r}}^{(M)}, a_{1}\right] v, v\right\rangle \leq C_{M}+\left(C_{0}+C_{1}+2\right)\left\|b_{\tilde{r}}^{(M)} v\right\|_{H^{0}}^{2} .
$$

Now fix $M \geq\left(C_{0}+C_{1}+2\right)$ and drop the $M$-indices. The a priori assumption on $v$ implies that the right-hand side in (3.8) is finite, so

$$
\operatorname{Re}\left\langle\left(\frac{1}{i} b_{\tilde{r}}^{*}\left[b_{\tilde{r}}, a_{1}\right]-M b_{\tilde{r}}^{*} b_{\tilde{r}}\right) v, v\right\rangle \leq C .
$$

For $e_{2 \tilde{r}}$ and $b_{2 \tilde{r}}$ as in (3.7), it follows from the hypotheses on $v$ (since $\left\langle b_{2 \tilde{r}} v, v\right\rangle \leq C$ ) that

$$
\left\langle e_{2 \tilde{r}}(x, D) v, v\right\rangle+\operatorname{Re}\left\langle\left(\frac{1}{i} b_{\tilde{r}}^{*}\left[b_{\tilde{r}}, a_{1}\right]-M b_{\tilde{r}}^{*} b_{\tilde{r}}+b_{2 \tilde{r}}-e_{2 \tilde{r}}\right)(x, D) v, v\right\rangle \leq C .
$$

By the calculus of smooth pseudodifferential operators,

$$
\begin{aligned}
& \frac{1}{i} \Lambda^{-\tilde{r}+\alpha / 2} b_{\tilde{r}}^{*}\left[b_{\tilde{r}}, a_{1}\right] \Lambda^{-\tilde{r}+\alpha / 2} \\
& \quad=b_{\tilde{r}+\alpha / 2}(x, D) a_{1-\tilde{r}+\alpha / 2}(x, D)-b_{\alpha / 2}(x, D) a_{1+\alpha / 2}
\end{aligned}
$$

where $b_{l} \in S_{1,0}^{l}, a_{l} \in S_{s t}^{l ; s+2 ; r+2}(\Gamma)$. From Theorem 1.10(ii), with $k=2$, and with $j=1, \mu=\tilde{r}+\alpha / 2-1, m=-1-\tilde{r}+\alpha / 2$ for $a_{1-\tilde{r}+\alpha / 2}$ and $j=0, \mu=\alpha / 2$, $m=-1+\alpha / 2$ for $a_{1+\alpha / 2}$, it follows that

$$
\begin{aligned}
& b_{\tilde{r}+\alpha / 2}(x, D) a_{1-\tilde{r}+\alpha / 2}(x, D)-b_{\alpha / 2}(x, D) a_{1+\alpha / 2} \\
& \quad=(\text { terms of order } 1+\alpha)+(\text { terms of order } \alpha)+r(x, D),
\end{aligned}
$$

where

$$
\begin{aligned}
r(x, D)= & a_{\alpha-1}(x, D)+p_{\tilde{r}+\alpha / 2-1}(x, D) a_{-\tilde{r}+\alpha / 2}(x, D) \\
& +p_{\alpha / 2}(x, D) a_{-1+\alpha / 2}(x, D) .
\end{aligned}
$$

Here $a_{l} \in S^{l ; s, r}(\Gamma), p_{l} \in S_{1,0}^{l}$. From (3.10), the terms of order $(1+\alpha)$ sum to zero and the terms of order $\alpha$ give

$$
\frac{1}{2} H_{a_{1}} b_{\tilde{r}}^{2}(x, \xi)\langle\xi\rangle^{-2 \tilde{r}+\alpha} \in S_{s t}^{\alpha ; s+1, r+1}(\Gamma) .
$$


Let $b_{0} \in S_{1,0}^{0}$ have support in $\Gamma, b_{0} \geq 0, b_{0} \equiv 1$ on $\operatorname{supp} b_{\tilde{r}} \cup \operatorname{supp} b_{2 \tilde{r}} \cup \operatorname{supp} e_{2 \tilde{r}}$. To estimate

$$
\left|\left\langle b_{0}(x, D) r(x, D) \Lambda^{\tilde{r}-\alpha / 2} v, b_{0}(x, D) \Lambda^{\tilde{r}-\alpha / 2} v\right\rangle\right|
$$

we write

$$
\begin{aligned}
r(x, D) \Lambda^{\tilde{r}-\alpha / 2}= & a_{\tilde{r}-1+\alpha / 2}(x, D)+p_{\tilde{r}+\alpha / 2-1}(x, D) a_{0}(x, D) \\
& +p_{\alpha / 2}(x, D) a_{-1+\tilde{r}}(x, D) .
\end{aligned}
$$

Since $r \geq \tilde{r}-1+\alpha / 2$, and $\tilde{r}-\alpha / 2 \geq \tilde{r}-1+\alpha / 2$, Theorem 1.3 and the hypothesis on $v$ are easily seen to imply that $\left|\left\langle b_{0} r \Lambda^{\tilde{r}-\alpha / 2} v, b_{0} \Lambda^{\tilde{r}-\alpha / 2} v\right\rangle\right| \leq C$. And from (3.10), (3.9), the calculus of smooth pseudodifferential operators, and the properties of $b_{0}$, we conclude that

$$
\begin{aligned}
\left\langle e_{2 \tilde{r}}(x, D) v, v\right\rangle+\operatorname{Re}\left\langle\left\{\frac{1}{2} H_{a_{1}} b_{\tilde{r}}^{2}(x, D) \Lambda^{-2 \tilde{r}+\alpha}\right.\right. & \\
\left.-\Lambda^{-\tilde{r}+\alpha / 2}\left(M b_{\tilde{r}}^{*} b_{\tilde{r}}+b_{2 \tilde{r}}-e_{2 \tilde{r}}\right) \Lambda^{-\tilde{r}+\alpha / 2}\right\} & \\
& \left.\times b_{0} \Lambda^{\tilde{r}-\alpha / 2} v, b_{0} \Lambda^{\tilde{r}-\alpha / 2} v\right\rangle \leq C
\end{aligned}
$$

since $H_{a_{1}} b_{\tilde{r}}^{2}(x, \xi)=H_{a_{1}} b_{\tilde{r}}^{2}(x, \xi) b_{0}(x, \xi)$. The operator in braces has symbol

$$
\left(\frac{1}{2} H_{a_{1}} b_{\tilde{r}}^{2}-M b_{\tilde{r}}^{2}+b_{2 \tilde{r}}-e_{2 \tilde{r}}\right)(x, \xi)\langle\xi\rangle^{-2 \tilde{r}+\alpha} \in S_{s t}^{\alpha ; s+1, r+1}(\Gamma) \subset S_{s t}^{\alpha ; n / 2+\delta+2 \alpha}(U)
$$

for small $\delta>0$, since $r \geq s$ and we can assume that $2 \alpha<s+1-n / 2$. Moreover, the principal symbol is nonnegative by (3.9). Since $b_{0}(x, D) \Lambda^{\tilde{r}-\alpha / 2} v \in H^{0}$ by the hypotheses on $v$, and we can assume that $b_{0}(x, \xi)$ has compact $x$-support, Lemma 3.1 and (3.11) imply that

$$
\left\langle e_{2} \tilde{r}(x, D) v, v\right\rangle \leq C .
$$

Thus the norm of $v$ in $H_{m l}^{\tilde{r}}(\gamma)$ depends only on the norms of $a, v$ and $f$ in the hypotheses.

The final linear propagation result to be considered, with local instead of microlocal hypotheses, treats the case where $s$ is near $n / 2$, and is the analogue of Theorem 2 in $[\mathbf{1}]$. For $a(x, \xi) \in S_{s t}^{m+2 ; s+1}(U)$ with real homogeneous principal symbol and $n / 2<s, \nabla_{x} a_{m+2} \in C^{\delta}$ for small $\delta>0$, so if $U$ is a small neighborhood of the characteristic point $\left(x_{0}, \xi_{0}\right)$ of $a_{m+2}$ there is a $C^{1+\delta}$ null bicharacteristic $\gamma$ (possibly not unique) of $a_{m+2}$ through $\left(x_{0}, \xi_{0}\right)$.

THEOREM 3.3. Let $n / 2<s \leq n / 2+1$. Assume that $a(x, \xi) \in S_{s t}^{m+2 ; s+1}(U)$ has real principal symbol $a_{m+2}$ homogeneous of degree $m+2$ and that the null bicharacteristic $\gamma$ through $\left(x_{0}, \xi_{0}\right)$ is unique. If

(i) $v \in H^{s+m}(U)$,

(ii) $v \in H_{m l}^{s+m+\varepsilon}\left(x_{0}, \xi_{0}\right)$ for some $\varepsilon<s-n / 2$,

(iii) $f \in H^{s}(U)$,

(iv) $a(x, D) v=f$, then $v \in H_{m l}^{s+m+\varepsilon}(\gamma)$.

ProOF. We follow the proof of Theorem 3.2, indicating the necessary modifications. Again replace $\gamma$ and $\left(x_{0}, \xi_{0}\right)$ with $\Gamma$ and $\Gamma_{0}$, and take $m=-1, a(x, \xi)=$ $a_{1}(x, \xi)+a_{0}(x, \xi)$; this time with $a_{j} \in S_{s t}^{j ; s+j}(U)$. If we define $e_{0}^{\delta}(\xi)$ as before, then by Lemma 1.12 with $k=2, m=-1, \mu=0$, we have

$$
e_{0}^{\delta}(D) a(x, D)=a_{1}(x, D) e_{0}^{\delta}(D)+\tilde{a}_{0, \delta}(x, D) e_{0}^{\delta}(D)+r_{\delta}(x, D)
$$


where $r_{\delta}: H_{\text {loc }}^{s-1}(U) \rightarrow H_{\text {loc }}^{s-1+\varepsilon}(U)$ and $\left\{\tilde{a}_{0, \delta}\right\} \subset S^{0 ; s}(U)$ uniformly in $0<\delta \leq 1$. We have used the fact that if $\varepsilon<\frac{1}{2}$, then $r_{\delta}(x, D) \in S^{-\varepsilon ; s-\varepsilon}(U): H_{\text {loc }}^{s-1}(U) \rightarrow$ $H_{\text {loc }}^{s-1+\varepsilon}(U)$, while if $\varepsilon>\frac{1}{2}, r_{\delta}(x, D)=p_{0}(x, D) r_{0}(x, D)$, where $r_{0} \in S^{-1+\varepsilon ; s-\varepsilon}(U)$ : $H_{\mathrm{loc}}^{s-1}(U) \rightarrow H_{\mathrm{loc}}^{s-\varepsilon}(U)$ and $p_{0} \in S_{1,0}^{1-2 \varepsilon}: H_{\mathrm{loc}}^{s-\varepsilon}(U) \rightarrow H_{\mathrm{loc}}^{s-1+\varepsilon}(U)$. As before it then follows that we may assume a priori that $v \in H_{\text {loc }}^{s-1+\varepsilon}(U)$, and that $a(x, D) v=f$ where $a(x, \xi) \in S_{s t}^{1 ; s+1}(U)$ and

(i) $v \in H^{s-1} \cap H^{\tilde{r}-\alpha / 2}(\Gamma)$,

(ii) $v \in H_{m l}^{\tilde{r}}\left(\Gamma_{0}\right)$,

(iii) $f \in H^{\tilde{r}}(U)$

for some $\alpha>0$ and $s-1+\alpha / 2 \leq \tilde{r} \leq s-1+\varepsilon$. We must show that $\|v\|_{H_{m l}^{\tilde{r}}(\gamma)}$ is bounded by a constant depending only on the norms of $a, v$ and $f$ in (i), (ii) and (iii).

We fix $\alpha<\frac{1}{2} \min (s-n / 2,1-\varepsilon)$. Exactly as before, since $a_{1} \in C^{1+\alpha}$, there are symbols $b_{\tilde{r}}^{(M)} \in S_{1,0}^{r}, b_{2 \tilde{r}}, e_{2 \tilde{r}} \in S_{1,0}^{2 \tilde{r}}$ supported on $\Gamma$, with $e_{2 \tilde{r}}$ elliptic on $\gamma$, such that

$$
\begin{gathered}
\frac{1}{2} H_{a_{1}}\left(b_{\tilde{r}}^{(M)}\right)^{2}-M\left(b_{\tilde{r}}^{(M)}\right)^{2}+b_{2 \tilde{r}} \geq e_{2 \tilde{r}} \\
\operatorname{Re} \frac{1}{i}\left\langle b_{\tilde{r}}^{(M)^{*}}\left[b_{\tilde{r}}^{(M)}, a_{1}\right] v, v\right\rangle=\operatorname{Im}\left\langle-b_{\tilde{r}}^{(M)} a_{0} v, b_{\tilde{r}}^{(M)} v\right\rangle+\operatorname{Im}\left\langle b_{\tilde{r}}^{(M)} f, b_{\tilde{r}}^{(M)} v\right\rangle \\
-\frac{1}{2 i}\left\langle\left(a_{1}-a_{1}^{*}\right) b_{\tilde{r}}^{(M)} v, b_{\tilde{r}}^{(M)} v\right\rangle
\end{gathered}
$$

The second and third terms on the right satisfy the same estimates as before, since $a_{1}$ is real and Theorem 1.10(iii) with $m=0$ and $k=1$ implies $\left(a_{1}-a_{1}^{*}\right) \in S^{0 ; s}(U)$. We have

$$
\left\langle b_{\tilde{r}}^{(M)} a_{0} v, b_{\tilde{r}}^{(M)} v\right\rangle=\left\langle a_{0} b_{\tilde{r}}^{(M)} v, b_{\tilde{r}}^{(M)} v\right\rangle+\left\langle\left[b_{\tilde{r}}^{(M)}, a_{0}\right] v, b_{\tilde{r}}^{(M)} v\right\rangle
$$

with the first term on the right estimated as above. And, from Lemma 1.12 with $\mu=\tilde{r}, k=1, m=-1$ and $\delta=\alpha / 2$, we have $\left[b_{\tilde{r}}^{(M)}, a_{0}\right]=c(x, D)$ where $c(x, D)=$ $c_{-\alpha / 2+\tilde{r}}(x, D)+p_{\tilde{r}}(x, D) c_{-\alpha / 2}(x, D)$ with $c_{l} \in S^{l ; s-\alpha / 2}(U)$ and $p_{\tilde{r}} \in S_{1,0}^{\tilde{r}}$. From Theorem 1.3 and the hypotheses on $v$ and $f$, if $b_{0} \in S_{1,0}^{0}$ has support in $\Gamma, b_{0} \equiv 1$ on $\operatorname{supp} b_{\tilde{r}}^{(M)}$, then

$$
\left|\left\langle b_{0}(x, D) c(x, D) v, b_{\tilde{r}}^{(M)} v\right\rangle\right| \leq C_{M}+\left\|b_{\tilde{r}}^{(M)} v\right\|_{H^{0}} .
$$

Thus we again have

$$
\operatorname{Re} \frac{1}{i}\left\langle b_{\tilde{r}}^{(M)^{*}}\left[b_{\tilde{r}}^{(M)}, a_{1}\right] v, v\right\rangle \leq C_{M}+\left(C_{0}+C_{1}+2\right)\left\|b_{\tilde{r}}^{(M)} v\right\|_{H^{0}}^{2},
$$

and so by the a priori assumption,

$$
\left\langle e_{2 \tilde{r}}(x, D) v, v\right\rangle+\operatorname{Re}\left\langle\left(i^{-1} b_{\tilde{r}}^{*}\left[b_{\tilde{r}}, a_{1}\right]-M b_{\tilde{r}}^{*} b_{\tilde{r}}+b_{2 \tilde{r}}-e_{2 \tilde{r}}\right)(x, D) v, v\right\rangle \leq C .
$$

Again

$$
\frac{1}{i} \Lambda^{-\tilde{r}+\alpha / 2} b_{\tilde{r}}^{*}\left[b_{\tilde{r}}, a_{1}\right] \Lambda^{-\tilde{r}+\alpha / 2}=\frac{1}{2} H_{a_{1}} b_{\tilde{r}}^{2}(x, D) \Lambda^{-2 \tilde{r}+\alpha}+r(x, D) .
$$

By Lemma 1.12 with $k=2, \delta=\alpha$, and the two cases $\{\mu=\tilde{r}+\alpha / 2, m=-1-\tilde{r}+$ $\alpha / 2\}$ and $\{\mu=\alpha / 2, m=-1+\alpha / 2\}$,

$$
\begin{aligned}
r(x, D) \Lambda^{\tilde{r}-\alpha / 2}= & c_{\tilde{r}-\alpha / 2}(x, D)+p_{\tilde{r}+\alpha / 2}(x, D) c_{-\alpha}(x, D) \\
& +p_{\alpha / 2}(x, D) c_{\tilde{r}-\alpha}(x, D)
\end{aligned}
$$


where $c_{l} \in S^{l ; s-\alpha}(U)$ and $p_{l} \in S_{1,0}^{l}$. Since $\tilde{r} \leq s-1+\varepsilon$, it follows that $s-\alpha \geq \tilde{r}+\alpha / 2$, and so Theorem 1.3 implies $r(x, D): H^{s-1} \cap H_{m}^{\tilde{r}-\alpha / 2}(\Gamma) \rightarrow H_{m l}^{0}(\Gamma)$. Thus for $b_{0}$ with support on $\Gamma$, by the hypotheses on $v$ and $f$,

$$
\left|\left\langle b_{0} r \Lambda^{\tilde{r}-\alpha / 2} v, b_{0} \Lambda^{\tilde{r}-\alpha / 2} v\right\rangle\right| \leq C .
$$

As before, it follows from (3.13) that

$$
\begin{aligned}
\left\langle e_{2 \tilde{r}}(x, D) v, v\right\rangle+\operatorname{Re}\left\langle\left\{\frac{1}{2} H_{a_{1}} b_{\tilde{r}}^{2}(x, D) \Lambda^{-2 \tilde{r}+\alpha}\right.\right. & \\
\left.-\Lambda^{-\tilde{r}+\alpha / 2}\left(M b_{\tilde{r}}^{*} b_{\tilde{r}}+b_{2 \tilde{r}}-e_{2 \tilde{r}}\right) \Lambda^{-\tilde{r}+\alpha / 2}\right\} & \\
& \left.\times b_{0} \Lambda^{\tilde{r}-\alpha / 2} v, b_{0} \Lambda^{\tilde{r}-\alpha / 2} v\right\rangle \leq C .
\end{aligned}
$$

The operator in braces has symbol in $S^{\alpha ; s}(U) \subset S^{\alpha ; n / 2+\delta+2 \alpha}(U)$ for small $\delta>0$ by the choice of $\alpha$. By (3.12) and Lemma 3.1 the a priori estimate holds.

4. Application to quasilinear hyperbolic problems. In this section we show how Theorems 2.1, 3.2 and 3.3 can be combined with a simple iteration argument to yield information about the propagation of weak singularities in quasilinear hyperbolic equations. We refer to the singularities as weak since we must assume that our solutions are $H^{\sigma}$ for some $\sigma>n / 2$. Therefore shocks are not permitted. Thus these results describe the propagation of weak singularities in regions away from shocks, or, if the data are relatively smooth, in all of $\mathbf{R}^{n}$ before the onset of shocks.

We will study quasilinear equations of order $\mu=m+2, m \geq-1$ :

$$
\sum_{|\alpha|=\mu} b_{\alpha}\left(x, u, D u, \ldots, D^{\mu-1} u\right) D^{\alpha} u(x)=f\left(x, u, D u, \ldots, D^{\mu-1} u\right) .
$$

We suppose that $b_{\alpha}(\cdot)$ and $f$ are $C^{\infty}$ functions and that we are given a function $u \in H_{\mathrm{loc}}^{s+\mu+1}(0)$ with $s>n / 2$ which satisfies (4.1) in an open set 0 . The symbols $D u, \ldots, D^{\mu-1} u$, indicate that $b_{\alpha}\left(x, u, D u, \ldots, D^{\mu-1} u\right)$ and $f\left(x, u, D u, \ldots, D^{\mu-1} u\right)$ may depend on all derivatives of $u$ up to order $\mu-1$. For $x \in O, \xi \in \mathbf{R}^{n} \backslash\{0\}$, let

$$
p(x, \xi)=\sum_{|\alpha|=\mu} b_{\alpha}(x) \xi^{\alpha} .
$$

Since $u \in H^{s+\mu+1}$, we have $p \in C^{2}$. Thus for each $x_{0} \in \mathcal{O}$ and $\xi_{0} \in \mathbf{R}^{n} \backslash\{0\}$ such that $p\left(x_{0}, \xi_{0}\right)=0$, the solution of $\dot{x}(t)=\nabla_{\xi} p(x, \xi), x(0)=x_{0}, \dot{\xi}(t)=-\nabla_{x} p(x, \xi)$, $\xi(0)=\xi_{0}$ is a well-defined bicharacteristic strip, at least for small $t$. let $\gamma$ denote a compact connected piece of this bicharacteristic strip containing $\left(x_{0}, t_{0}\right)$ so that the projection of $\gamma$ onto its first coordinates lies entirely within 0 . We know by hypothesis that $u \in H^{s+\mu+1}(0)$. The question we wish to answer is this: suppose that, in addition, we are given that $u \in H_{m l}^{r+\mu+2}\left(p_{0}\right)$ at $p_{0}=\left(x_{0}, \xi_{0}\right) \in \gamma$ for some $r \geq s$. When will it be true that $u \in H_{m l}^{r+\mu+2}$ at $p_{1}$ for all $p_{1} \in \gamma$ ?

We cannot apply Theorem 3.2 directly to (4.1) because the coefficients $b_{\alpha}$ and $f$ are not smooth enough compared to $u$. We get around this difficulty by differentiating the equation. Let $\partial$ be a first order constant coefficient partial differential operator such that $p_{0} \notin$ Char $\partial$. Then $p \notin$ Char $\partial$ for all $p \in \mathcal{N} \cap \gamma$ where $\mathcal{N}$ is a neighborhood of $p_{0}$. If we can propagate improved regularity along $\mathcal{N} \cap \gamma$, then, by piecing together finitely many such arcs we can propagate along all of $\gamma$. Thus we 
may assume without loss that $p \notin$ Char $\partial$ for all $p \in \gamma$. We now apply $\partial$ to (4.1) three times, obtaining successively equations of the form

$$
\begin{aligned}
& \sum b_{\alpha}\left(D^{\mu-1} u\right) D^{\alpha} \partial u=g_{1}\left(D^{\mu} u\right), \\
& \sum b_{\alpha}\left(D^{\mu-1} u\right) D^{\alpha} \partial^{2} u=g_{2}\left(D^{\mu} u\right) D^{\mu+1} u, \\
& \sum b_{\alpha}\left(D^{\mu-1} u\right) D^{\alpha} \partial^{3} u=g_{3}\left(D^{\mu} u\right) D^{\mu+2} u+g_{4}\left(D^{\mu+1} u\right) .
\end{aligned}
$$

The expressions on the right sides of (4.2)-(4.4) stand for sums of $C^{\infty}$ functions of $\left\{D^{\beta} \partial^{\delta} u\right\}$ which have the orders of derivatives indicated. The point is that the highest order terms on the right side of (4.4) are linear in $D^{\mu+2} u$ and have coefficients of the form $g_{3}\left(D^{\mu} u\right)$. Thus (4.4) may be written

$$
\begin{gathered}
\sum_{|\alpha|=\mu} b_{\alpha}\left(D^{\mu-1} u\right) D^{\alpha} \partial^{3} u+\sum_{|\alpha|=\mu}\left\{h_{\alpha}\left(D^{\mu-1} u\right) D^{\alpha} \partial^{2} u+k_{\alpha}\left(D^{\mu-1} u\right) D^{\mu-1} \partial^{3} u\right\} \\
=l\left(D^{\mu+1} u\right)
\end{gathered}
$$

where $h_{\alpha}, k_{\alpha}, l$ are $C^{\infty}$ functions and $l\left(D^{\mu+1} u\right)$ is a shorthand way of writing $l\left(x, u, \ldots, D^{\mu+1} u\right)$. In differentiating the equations the many uses of the chain rule and Leibnitz rule are legitimate since the hypothesis that $u \in H^{s+\mu+1}$ guarantees that in each term at most one factor is in $H^{\sigma}$ for $\sigma \leq n / 2$. As usual, let $\Lambda=$ $(I-\Delta)^{1 / 2}$, and set $w=\Lambda^{3} u$ and $P_{0}=\partial^{3} \Lambda^{-3}$. Then $w$ satisfies $a(x, D) w=l$ where

$$
a(x, D)=\left\{\sum_{|\alpha|=\mu} b_{\alpha}\left(D^{\mu-1} u\right) D^{\alpha}\right\} P_{0}+\sum_{\beta} h_{\beta}\left(D^{\mu} u\right) Q_{\beta} .
$$

Here $Q_{\beta}$ are classical pseudodifferential operators of order $\mu-1$, and $P_{0}$ is a classical pseudodifferential operator of order zero which is elliptic in a neighborhood of $\gamma$. We can now use Theorem 3.2. Set $r=s$. Then,

$$
\begin{gathered}
w \in H^{s+m} \cap H_{m l}^{r+m}(\gamma), \\
b_{\alpha}\left(D^{\mu-1} u\right) \in H^{s+2} \cap H_{m l}^{r+2}(\gamma), \\
h_{\beta}\left(D^{\mu} u\right) \in H^{s+1} \cap H_{m l}^{r+1}(\gamma),
\end{gathered}
$$

so $a(x, D) \in S_{s t}^{m+2 ; s+2, r+2}$. Since

$$
a(x, D) w=l\left(D^{\mu+1} u\right) \in H^{s} \cap H_{m l}^{r}(\gamma)
$$

all the hypotheses on $a(x, D)$ in Theorem 3.2 are fulfilled. Thus, since we know that $u \in H_{m l}^{r+\mu+2}\left(p_{0}\right)$ for some $p_{0} \in \gamma$ and some $r \geq s$, then $w \in H_{m l}^{s+m+1}\left(p_{0}\right)$ and we conclude from Theorem 3.2 that

$$
w \in H^{s+m} \cap H_{m l}^{s+m+1}(p) \quad \forall p \in \gamma
$$

and

$$
u \in H^{s+\mu+1} \cap H_{m l}^{s+\mu+2}(p) \quad \forall p \in \gamma .
$$

Thus we have propagated some improved microlocal regularity of $u$ along $\gamma$. By (4.9) and Rauch's Lemma (as in Theorem 1.3), if $r \leq \min (s+1,2 s-n / 2-\varepsilon$ ),

$$
\begin{aligned}
b_{\alpha}\left(D^{\mu-1} u\right) & \in H^{s+2} \cap H_{m l}^{r+2}(\gamma), \\
h_{\beta}\left(D^{\mu} u\right) & \in H^{s+1} \cap H_{m}^{r+1}(\gamma), \\
l\left(D^{\mu+1} u\right) & \in H^{s} \cap H_{m l}^{r}(\gamma) .
\end{aligned}
$$


Finally,

$$
w \in H_{m l}^{r+m+1}\left(p_{0}\right) .
$$

Thus, the hypotheses of Theorem 3.2 are fulfilled so we conclude that

$$
w \in H_{m l}^{r+m+1}(\gamma), \quad u \in H_{m l}^{r+\mu+2}(\gamma) .
$$

Now we can use Rauch's Lemma again to improve the microlocal regularity of the coefficients and then use Theorem 3.2 again, as long as $r<2 s-n / 2$. We have thus proven the following theorem in the case $\sigma=s+1>n / 2+1$.

THEOREM 4.1. Let $O$ be an open region of $\mathbf{R}^{n}$, and let $n / 2<\sigma \leq \rho<$ $2 \sigma-n / 2$. For $x_{0} \in \mathcal{O}$, suppose that $p_{0}=\left(x_{0}, \xi_{0}\right)$ is on a null bicharacteristic $\gamma$ of $\sum_{|\alpha|=\mu} b_{\alpha}\left(x, u, \ldots, D^{\mu-1} u\right) D^{\alpha}$. If $u \in H^{\sigma+\mu}(0)$ satisfies (4.1) and $u \in H_{m l}^{\rho+\mu}\left(p_{0}\right)$, then $u \in H_{m l}^{\rho+\mu}(p)$ for all $p \in \gamma$.

REMARK. If $\sigma>n / 2+1$, the null bicharacteristic strip is unique. If $n / 2<\sigma \leq$ $n / 2+1$, we assume that it is unique.

PROOF. It remains to handle the case where $n / 2<\sigma \leq n / 2+1$. In this case we may not differentiate the equation three times since that would result in products of the form $\phi_{1} \phi_{2}$ where $\phi_{i} \in H^{s}$ for $s<n / 2$. We can, however, differentiate with $\partial$ twice to obtain

$$
\sum_{|\alpha|=\mu} b_{\alpha}\left(D^{\mu-1} u\right) D^{\alpha} \partial^{2} u+h_{\alpha}\left(D^{\mu} u\right) D^{\alpha} \partial u+k_{\alpha}\left(D^{\mu} u\right) D^{\mu-1} \partial^{2} u=l\left(D^{\mu} u\right)
$$

If we now set $w=\Lambda^{2} u$ and $P_{0}=\partial^{2} \Lambda^{-2}$, then $w$ satisfies

$$
\sum_{|\alpha|=\mu} b_{\alpha}\left(D^{\mu-1} u\right) D^{\alpha} P_{0} w+\sum h_{\beta}\left(D^{\mu} u\right) Q_{\beta} w=l\left(D^{\mu} u\right)
$$

where the $Q_{\beta}$ are classical pseudodifferential operators of order $\mu-1$. Since $u \in$ $H^{\sigma+\mu}$, we have $D^{\mu} u \in H^{\sigma}$, so $b_{\alpha}\left(D^{\mu-1} u\right) \in H^{\sigma+1}$ and $h^{\alpha}\left(D^{\mu} u\right), l\left(D^{\mu} u\right) \in H^{\sigma}$. Set $m=\mu-2$ and $\varepsilon=\rho-\sigma<\sigma-n / 2$. then

$$
\sum_{|\alpha|=\mu} b_{\alpha}\left(D^{\mu-1} u\right) D^{\alpha} P_{0}+\sum h_{\beta}\left(D^{\mu} u\right) Q_{\beta} \in S_{s t}^{m+2 ; \sigma+1}
$$

so the hypotheses of Theorem 3.3 are satisfied with $\sigma$ replacing $s$. Therefore $w \in$ $H_{m l}^{\sigma+m+\varepsilon}(p)$ for all $p \in \gamma$, which yields $u \in H_{m l}^{\rho+\mu}(p)$ for all $p \in \gamma$.

EXAMPLE 1 (THE QUASILINEAR WAVE EQUATION). Consider the equation

$$
u_{t t}-\sum b_{i j}(\nabla u) \partial_{i} \partial_{j} u=0 .
$$

Suppose that (4.13) has a solution $u \in H^{\sigma+2}, \sigma>n / 2$, in a region $R$ whose domain of dependence contains a ball $B$ at $t=0$ (see Figure 4.1). We suppose that $\sum b_{i j}(\nabla u) \xi_{i} \xi_{j} \geq c|\xi|^{2}$ and that the initial data are in $H^{\sigma+2}$ overall, but $C^{\infty}$ outside of $B$. What further regularity properties of $u$ can we derive? Since $\nabla u$ is $C^{1}$, there is a well-defined forward characteristic hypersurface emanating from each point of $B$. On the union of these hypersurfaces, denoted by II in Figure 4.1, we do not expect the solution to be better than $H^{\sigma+2}$. In region I the solution is $C^{\infty}$ by finite propagation speed. Consider a point $x_{1}$ in region III. By applying Theorem 
4.1 on the backward bicharacteristic curves from $x_{1}$ (for example, from $x_{1}$ to $x_{0}$ ), we see that

$$
u \in H^{\sigma+2} \cap H_{m l}^{2 \sigma-n / 2+2-\varepsilon}\left(x_{1}, \xi_{1}\right)
$$

for any characteristic direction $\xi_{1}$ at $x_{1}$ and any $\varepsilon>0$.

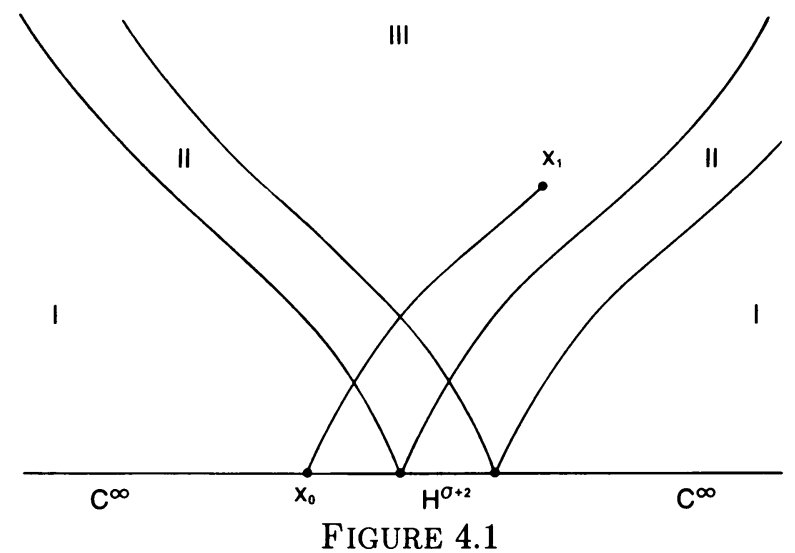

Now suppose that $\xi_{2}$ is a noncharacteristic direction at $x_{1}$.

Differentiate once in (4.13), as in the proof of Theorem 4.1, to obtain

$$
a(\nabla u) D^{2} \partial u=b\left(D^{2} u\right) \text {. }
$$

Set $w=\Lambda u \in H^{\sigma+1}$; then $a(x, D) w=f$, where $a \in S_{s t}^{1+1 ; \sigma+1}$ and $f \in H^{\sigma}$. So Theorem 2.2 implies that $w \in H^{\sigma+1} \cap H_{m l}^{\sigma+2}\left(x_{1}, \xi_{2}\right)$, and hence

$$
u \in H^{\sigma+2} \cap H_{m l}^{\sigma+3}\left(x_{1}, \xi_{2}\right) .
$$

Since this is true for any elliptic direction $\xi_{2},(4.15)$ and (4.14) imply that $u \in$ $H^{\min \{\sigma+3,2 \sigma-n / 2+2-\varepsilon\}}$ at $x_{1}$. If $\sigma+3<2 \sigma-n / 2+2-\varepsilon$ we can simply apply the above procedure again to conclude that $u \in H^{\sigma+3} \cap H_{m l}^{\sigma+4}\left(x_{1}, \xi_{2}\right)$ for elliptic directions $\xi_{2}$. We can continue to apply Theorem 2.1 to improve the regularity in elliptic directions until it is better than the regularity in the characteristic directions, so we find that $u \in H^{2 \sigma-n / 2+2-\varepsilon}$ at $x_{1} \in$ region III for all $\varepsilon>0$.

In the linear case, $u$ would be $C^{\infty}$ in region III. In the quasilinear case, the interaction of the singularities on the characteristic hypersurfaces in region II produces, in general, new weak singularities which propagate into region III. We have shown that these new singularities have strength at most $H^{2 \sigma-n / 2+2-\varepsilon}$.

EXAMPLE 2 (A COUPLED ELLIPTIC-HYPERBOLIC SYSTEM). We will show how to use Theorems 2.1, 4.1, and Rauch's Lemma to derive regularity results for the system

$$
\begin{gathered}
\sum a_{i j}(u) \partial_{i} \partial_{j} v=g(u, v), \\
u_{t t}-\sum b_{i j}(v) \partial_{i} \partial_{j} u=f(u, v) .
\end{gathered}
$$

We suppose that $a_{i j}, b_{i j}, g, f$ are $C^{\infty}$ functions and that $u$ and $v$ are $H^{\sigma+1}$ solutions of (4.16), (4.17), $\sigma>n / 2$, in a region $R$ as in Example 1 and Figure 4.1. We suppose that

$$
\begin{aligned}
& \sum a_{i j}(u) \xi_{i} \xi_{j} \geq c\left(1+|\xi|^{2}\right), \\
& \sum b_{i j}(v) \xi_{i} \xi_{j} \geq c\left(1+|\xi|^{2}\right)
\end{aligned}
$$


in $R$ so that (4.16) and (4.17) are elliptic and hyperbolic, respectively. As in Example 1, we assume that the data at $t=0$ for $(4.17)$ is $H^{\sigma+1}$ overall and $C^{\infty}$ outside the ball $B$, and ask what improved regularity for $u$ and $v$ holds.

In region II, the union of forward light "cones" from $B$, we do not expect $u$ to be better than $H^{\sigma+1}$; by Theorem 2.2, $v$ is in $H^{\sigma+3}$ there. In region I, both $u$ and $v$ must be $C^{\infty}$ by the following simple iteration argument: First improve $v$ locally using (4.16) and Theorem 2.2; then improve $u$ locally by using (4.17) and a domain of dependence argument (or as in Example 1); now improve $v$ again and so forth.

Let $x_{1}$ be a point in region III and let $\xi_{1}$ be a characteristic direction for (4.17) at $x_{1}$. Let $\gamma$ be the backward bicharacteristic strip through $\left(x_{1}, \xi_{1}\right)$; the corresponding bicharacteristic curve from $x_{0}$ to $x_{1}$ is depicted in Figure 4.1. We improve the microlocal regularity of $u$ and $v$ on $\gamma$ by a three-step process: suppose by induction that $u \in H^{\sigma+1} \cap H_{m l}^{\rho+1}(\gamma)$ and $v \in H^{\sigma+3} \cap H_{m l}^{\rho+2}(\gamma)$, where $\rho<2 \sigma-n / 2$.

(i) We improve the microlocal regularity of $v$ on $\gamma$ by using (4.16) and Theorem 2.1 (the microlocal elliptic theorem) twice. Differentiation of (4.16) yields $a(x, D) w=h$, where $w=\Lambda^{2} v \in H^{\sigma} \cap H_{m l}^{\rho}(\gamma), a \in S_{s t}^{1 ; \sigma+1, \rho+1}, h \in H^{\sigma} \cap H_{m l}^{\rho}(\gamma)$. Hence $w \in H_{m l}^{\rho+1}(\gamma)$, and $v \in H^{\sigma+3} \cap H_{m l}^{\rho+3}(\gamma)$.

(ii) Because of (i) and Rauch's Lemma, $b_{i j}(v)$ has been improved along $\gamma$. We can therefore improve the microlocal regularity of $u$ on $\gamma$ by using Theorem 3.2 (the microlocal hyperbolic propagation theorem) and (4.17). We have $b(x, D) u=f$, where $b \in S_{s t}^{2 ;(\sigma+1)+2,(\rho+1)+2}, u, f \in H^{\sigma+1} \cap H_{m l}^{\rho+1}(\gamma)$, and therefore $u \in H^{\sigma+1} \cap$ $H_{m l}^{\rho+2}(\gamma)$.

(iii) Since $u$ and $v$ have both been improved along $\gamma$, we can use Rauch's Lemma to improve $f(u, v), g(u, v)$ and $a_{i j}(u)$.

Since $a_{i j}(u)$ and $g(u, v)$ are improved on $\gamma$ we can return to (i) and improve $v$ further. We can continue this process until it is no longer possible to improve $a_{i j}(u), f, g$ because the hypotheses of Rauch's Lemma are no longer satisfied. We conclude that

$$
u \in H^{\sigma+1} \cap H_{m l}^{2 \sigma-n / 2+2-\varepsilon}(\gamma), \quad v \in H^{\sigma+3} \cap H_{m l}^{2 \sigma-n / 2+3-\varepsilon}(\gamma)
$$

for all $\varepsilon>0$.

Suppose now that $\xi_{2}$ is a noncharacteristic direction for (4.17) at $x_{1}$. We then improve $u$ and $v$ microlocally at $\left(x_{1}, \xi_{2}\right)$ by using the equations $(4.16),(4.17)$ and Theorem 2.1 as we did in Example 1:

$$
\begin{aligned}
& u \in H^{\sigma+1} \cap H^{2 \sigma-n / 2+2-\varepsilon}\left(x_{1}, \xi_{2}\right), \\
& v \in H^{\sigma+3} \cap H^{2 \sigma-n / 2+3-\varepsilon}\left(x_{1}, \xi_{2}\right) .
\end{aligned}
$$

From (4.18) and (4.19) we have

$$
\begin{array}{ll}
u \in H^{2 \sigma-n / 2+2-\varepsilon} & \text { at } x_{1}, \\
v \in H^{2 \sigma-n / 2+3-\varepsilon} & \text { at } x_{1}
\end{array}
$$

for all $\varepsilon>0$.

Notice that it is important in this example that the hypotheses of the elliptic regularity theorem permit microlocal hypotheses on the coefficients. The coefficient $a_{i j}(u)$ will never be better than $H^{\sigma}$ locally in region II. But it can be improved microlocally which allows us to improve $v$ microlocally and so forth. We remark 
that we have treated the case $a_{i j}=a_{i j}(u)$ and $b_{i j}=b_{i j}(v)$ for the sake of simplicity. The more general case $a_{i j}=a_{i j}(u, D u, v, D v), b_{i j}=b(u, D u, v, D v)$ can be handled by differentiating the equations as in Example 1.

\section{BIBLIOGRAPHY}

1. M. Beals and M. Reed, Propagation of singularities for hyperbolic pseudodifferential operators with nonsmooth coefficients, Comm. Pure Appl. Math. 35 (1982), 169-184.

2. M. Beals, C. Fefferman and R. Grossman, Strictly pseudoconvex domains in $C^{n}$, Bull. Amer. Math. Soc. (N.S.) 8 (1983), 125-322.

3. J.-M. Bony, Calcul symbolique et propagation des singularitées pour les équations nonlinéaires, Exposé XXII, Séminaire Goulaouic-Schwartz, Ecole Polytechnique, Paris, 1980.

4. $\ldots$, Calcul symbolique et propagation des singularitées pour les équations aux dérivées partielles non linéaires, Ann. Sci. École Norm. Sup. (4) 14 (1981), 209-246.

5. Shu Xing Chen, Pseudo-differential operators with finitely smooth symbol and their application to quasi-linear equations, Nonlinear Anal. 6 (1982), 1193-1206.

6. D. Gilbarg and N. S. Trudinger, Elliptic partial differential equations of second order, SpringerVerlag, Berlin, 1977.

7. L. Hörmander, Linear differential operators, Actes Congr. Internat. Math., vol. 1 (Nice, 1970), Gauthier-Villars, Paris, 1971, pp. 121-133.

8. _, On the extistence and regularity of solutions of linear pseudo-differential equations, Enseign. Math. (2) 17 (1971), 99-163.

9. L. Nirenberg, Lectures on linear partial differential equations, CBMS Regional Conf. Ser. in Math., no. 17, Amer. Math. Soc., Providence, R.I., 1973.

10. J. Rauch, Singularities of solutions to semilinear wave equations, J. Math. Pures Appl. 58 (1979), 299-308.

11. J. Rauch and M. Reed, Jump discontinuities of semilinear strictly hyperbolic systems in two variables: creation and propagation, Comm. Math. Phys. 81 (1981), 203-227.

12. __ Nonlinear microlocal analysis of semilinear hyperbolic systems in one space dimension, Duke Math. J. 49 (1982), 397-475.

13. __ Striated solutions of semilinear, two speed wave equations, preprint, 1984.

14. M. Taylor, Pseudo-differential operators, Princeton Univ. Press, Princeton, N.J., 1981.

Department of Mathematics, RUtgers University, New BRUNSWick, NeW JeRSEY 08903

Department of Mathematics, Duke University, Durham, North Carolina 27706 